\title{
La Carcinología argentina: una reseña histórica
}

\author{
Enrique E. Boschi \\ Consejo Nacional de Investigaciones Científicas y Técnicas \\ Instituto Nacional de Investigación y Desarrollo Pesquero \\ y Universidad de Buenos Aires Casilla 175, 7600 Mar del Plata, Argentina
}

RESUMEN. El documento describe el desarrollo de la Carcinología argentina, a partir de los primeros trabajos realizados por C. Berg en 1901. Al mismo tiempo, se incluye una revisión bibliográfica de trabajos realizados en la Argentina y efectuados por especialistas extranjeros sobre la fauna carcinológica de ese país.

Palabras claves: carcinología, historia, Argentina.

\section{Carcinology in Argentine: an historical review}

\begin{abstract}
The paper describes the carcinological development in Argentine since C. Berg 1901 paper's. A bibliographic review of foreing specialists who have published on carcinological fauna from Argentine is also included.
\end{abstract}

Key words: carcinology, history, Argentina.

\section{Los primeros pasos}

Los estudios carcinológicos en la Argentina no comienzan como en otras naciones de América con la presencia de algún destacado especialista extranjero, que al radicarse en el país, impulsa o a veces inicia los estudios en alguna disciplina. De aquellos sabios europeos, con extraordinarios conocimientos sobre las Ciencias Naturales, que llegaron al país entre fines y principios de siglo y asentaron las bases del conocimiento en geología, botánica y zoología en nuestro territorio, ninguno fue específicamente carcinólogo, aunque varios de ellos incursionaron muy puntualmente en el estudio de los crustáceos, como es el caso de Berg (1900) con la descripción de un notostraco, un estomatópodo y la mención de Blepharipoda, para las costas de Mar del Plata. Posteriormente, Berg (1901) se refiere al langostino y al camarón de la costa atlántica, que habían sido descriptos por Bate (1888) para el Atlántico sudoeste en la obra de la expedición del Challenger. Por otra parte existe la mención de Bruch (1916), destacado entomólogo, de dos especies de Branchiopoda de la provincia de San Luis y algunos otros trabajos con citas y descripciones de crustáceos, como los de Pesta sobre copépodos, Lahille y Doello Jurado sobre cirripedios.

Las expediciones oceanográficas de otros países al Atlántico Sudoccidental

Merecen ser mencionadas, al comienzo de esta reseña, por el significado que han tenido las grandes expediciones por el Atlántico Sur, a fines del siglo pasado y principios de éste, por las importantes colectas de material biológico y que han sido la base de inventarios de la fauna y de la flora marinas, en muchos casos muy poco conocidas, entre los que aparecen los crustáceos. Por ejemplo, la del H.M.S. «Challenger», la «Deutsche Sudpolar Expedition», la del «Discovery», etc. Se pueden destacar las campañas más recientes del B/I «Vema» de la Columbia University que realizó una serie de cruceros en el Atlántico Sudoeste entre 1953-1961, conducidos por el profesor M. Ewing del Lamont Geological Observatory. El primer volumen del Vema Reports, 1962, se refiere a crustáceos abisales (anfípodos, isópodos y cumáceos) colectados en las campañas del mencionado barco, siendo los autores tres autoridades en sus especialidades: Barnard (1962), Menzies (1962) y Bacescu (1962), respectivamente. 
El famoso navío francés «Calypso», realizó una campaña a la costa Atlántica de Sudamérica, entre 1961 y 1962, obteniendo importantes colecciones de organismos marinos, sobre los que aparecieron valiosos trabajos dedicados a crustáceos. También se puede citar los trabajos biológicos producidos por la «Soviet Antarctic Expedition» 1955-1958 (Vol. 4, 1970), que trata temas sobre cirripedios, cumáceos, isópodos de profundidad y decápodos de las regiones antártica y subantártica (Andriyashev y Ushavok, 1970). Varias otras campañas rusas recorrieron esta región realizando observaciones oceanográficas, pesqueras y recolectando material, así como de otras naciones, motivo por lo que resulta imposible citar a todas ellas.

Ultimamente se pueden mencionar las campañas del B/I «Meteor» de Alemania Federal, en diciembre de 1984, que en un viaje conjunto con científicos de Brasil, Uruguay y Argentina recorrió una amplia zona desde el sur de Brasil hasta el norte de la Patagonia argentina, señalando tres áreas especiales de estudios oceanográficos y biológicos, obteniendo valioso material de crustáceos planctónicos. Por parte del Instituto Nacional de Investigación y Desarrollo Pesquero (INIDEP) participaron M. Ehrlich, R. Sánchez y H. Mianzán.

\section{Los trabajos pioneros}

Desde principios de siglo en adelante, aparecen pocos trabajos elaborados en el país, pudiéndose indicar los ya mencionados de Berg entre otros autores, como se indicó anteriormente. Otra etapa se abre cuando el investigador argentino, profesor Birabén de la Facultad de Ciencias Naturales y Museo de La Plata, a partir de 1917, inicia el estudio de los cládoceros de aguas continentales (Birabén, 1917, 1918, 1939, 1954), intercalando también trabajos sobre anostracos a partir de 1946 (Birabén, 1946, 1951, 1957). Se debe señalar asimismo los aportes pioneros de Giambiagi (1922) y otros sobre isópodos, continuados y actualizados cincuenta años más tarde por Reca (1970, 1972, 1973).

Es oportuno destacar la aparición de la obra de Rathbun, del United States National Museum, que en una serie de cuatro voluminosos tomos trata los Brachyura de ambas costas del continente americano, basados en las colecciones del mencionado Museo y en otras importantes colecciones existentes en los Estados Unidos. Esta obra incluye gran parte de las especies marinas de este infraorden mencionadas para el litoral argentino (Rathbun, 1918, 1925, 1930, 1937).

\section{El impulso de los investigadores extranjeros}

En los años treinta hubo pocos avances en la carcinología argentina, pudiéndose mencionar los trabajos sobre copépodos de Wright (1927, 1938, 1939), sin olvidar la contribución de Brian (1925) de Italia, que estudió algunas muestras de este mismo grupo obtenidas por Silvestri. A partir de los años cuarenta y siguientes, se destaca el trabajo de los Anomura recientes del género Aegla, endémico para Sudamérica, realizado por Schmitt (1942a), carcinólogo del United States National Museum, Washington, quien realizó una revisión del grupo, sobre la base del material disponible en aquella época en instituciones argentinas y americanas y de algunas colecciones personales. El mismo especialista describe Blepharipoda doelloi única especie de Albuneidae existente en el litoral bonaerense (Schmitt, 1942b), citada por Berg como B. occidentalis. Brehm, de la Academia Austríaca de Ciencias, a partir de 1954 y hasta 1965, estudia los copépodos de aguas continentales de Argentina, con material remitido por Birabén (Brehm, 1954).

\section{El gran maestro. Los resultados de investigacio- nes en el propio país}

Por otra parte, comienzan a aparecer en la década del cuarenta las contribuciones sobre Aegla realizadas por el destacado zoólogo Ringuelet (1948a, 1948b, 1949a) profesor de Zoología de la Facultad de Ciencias Naturales y Museo de la Universidad Nacional de La Plata. Si bien Ringuelet, no trabajó sólo en crustáceos, sus contribuciones sobre estos invertebrados de aguas continentales han sido fundamentales en la Argentina, incluyendo los copépodos (Ringuelet, 1958a, 1958b, 1968; Ringuelet y Martínez, 1967, Cicchino y Ringuelet, 1977), los argúlidos (Branchiura) parásitos de peces (Ringuelet, 1943-1948), y los cangrejos de agua dulce de la familia Trichodactylidae, camarones palemónidos y sergéstidos (Ringuelet, 1949b), anfípodos de aguas dulces subterráneas (Grosso y Ringuelet, 1979), así como la descripción de un copépodo parásito (Ringuelet, 1945), entre otros, aparte de sus innumerables trabajos en los más diversos grupos zoológicos, lo que pone en evidencia el amplio conocimiento del reino animal que poseía este destacado especialista argentino, maestro de varias generaciones de biólogos, que lo constituye en uno de los más sobresalientes zoólogos contemporáneos del continente americano.

Posteriormente, Lopretto $(1978,1980)$ complementa los trabajos sobre los Aegla con el estudio 
del quinto pereiópodo de especies argentinas. Martin y Abele (1988) realizan un detallado examen de la morfología externa de especies del mencionado género de cangrejos anomuros.

Olivier (1954a) redescribe dos especies de cladóceros marinos para el litoral de Argentina, siguiendo el criterio de los autores extranjeros (Ramner, 1933) que previamente citaron estas especies para el Atlántico Sudoeste. El mismo Olivier (1954b) describe una nueva especie de cladócero de agua dulce del género Moina y posteriormente realiza una revisión de los Cladocera de la Argentina (Olivier, 1962). Ramírez (1981a) trata los Cladocera marinos del Atlántico Sudoccidental y, últimamente, Paggi (1995) ha estudiado exhaustivamente las formas continentales de Argentina.

Cicchino (1972, 1975) se ocupa del desarrollo postembrionario de varias especies de copépodos. En los últimos años investigan los copépodos de agua dulce Menú-Marque (1982), quien comenzara con estudios de desarrollo larval de decápodos marinos, Battistoni (1995) y Paggi (1994) que realizan un excelente trabajo revisando las especies neotropicales de Notodiaptomus. Cabe mencionar también los trabajos de Taberner (1988) sobre isópodos de agua dulce y los de Grosso y Claps (1984) y otros investigadores sobre isópodos y anfípodos del nordoeste de Argentina.

\section{La iniciación de los estudios biológico- pesqueros de crustáceos}

A partir de 1954 en el Departamento de Investigaciones Pesqueras, del entonces Ministerio de Agricultura y Ganadería de la Nación comenzaron, conducidos por Angelescu, los estudios cuantitativos del principal peneido de Mar del Plata (Pleoticus muelleri), mediante muestreos mensuales, que abarcaron un período de tres años apareciendo en 1959 los primeros resultados (Angelescu y Boschi, 1959) y como complemento se concretó un estudio morfológico de esta especie (Boschi y Angelescu, 1962).

Los primeros antecedentes sobre los peneidos de interés pesquero del mar Argentino, aparte de la descripción original, realizada por Bate (1888) y publicada en el tomo 24 de la Expedición del «Challenger» con una excelente impresión y un tomo especial de magníficas ilustraciones, ha sido la mención de Berg (1901), como se ha dicho, de ambas especies (Pleoticus muelleri y Artemesia longinaris) para las costas argentinas y uruguayas y los estudios morfológicos de las mismas, realizados por Fesquet (1933, 1941a, 1941b).

\section{La formación de un grupo de trabajo en Mar del Plata}

A partir de los años sesenta y con la experiencia adquirida al lado de Angelescu, iniciador de la biología pesquera en la Argentina, Boschi continuó con los estudios de los camarones palemónidos de agua dulce y los decápodos marinos en general, abarcando aspectos del desarrollo larval, inventario de las especies, distribución, cultivos experimentales, biología pesquera de los peneidos comerciales. A partir de esos primeros trabajos, se formó un grupo de estudios sobre los decápodos marinos en el ex-Instituto de Biología Marina de Mar del Plata, siendo los primeros colaboradores M.A. Scelzo, S. MenúMarque, B. Goldstein y J.L. Fenucci, aparte de un núcleo de estudiantes de la Universidad Nacional de La Plata y de la Universidad de Buenos Aires que se orientaron sobre estos estudios como J.H. Vinuesa, M. Muller, A.M. Petriella, J.C. Mallo, E.D. Spivak, R. Bacardit, V. Vera, entre otros y P. Cervellini de Bahía Blanca, así como jóvenes biólogos de distintas naciones latinoamericanas que llegaron al Instituto de Mar del Plata para adquirir experiencia en el estudio de estos invertebrados, que sería largo y difícil de enumerar. En la última década y con el Instituto Nacional de Investigación y Desarrollo Pesquero de Mar del Plata se incorporaron a estudios más aplicados sobre decápodos de interés pesquero M.I. Iorio, D. Bertuche, J. Wyngaard, C. Fischbach y M. Fernández.

\section{Estudio de la colección de decápodos del «Calypso»}

El material de crustáceos decápodos pagúridos obtenido con el «Calypso» en el viaje por el Atlántico Sudoccidental, durante 1961 y 1962, comprendió desde Recife (Brasil) hasta Mar del Plata (Argentina) y fue estudiado por dos distinguidos carcinólogos franceses, J. Forest y M. de Saint Laurent, el primero de los cuales participó en la campaña y visitó la Argentina. Los resultados fueron publicados en los Anales del Instituto Oceanográfico de París en 1967. Este trabajo constituye un importante aporte al estudio de los cangrejos ermitaños de esta región, así como las consideraciones zoogeográficas y afinidades que los autores han efectuado comparándolos con otras regiones vecinas. En esta misma serie los porcelánidos son estudiados por Haig (1966) de la Allan Hancock Foundation.

Manning (1966), el gran especialista mundial en Stomatopoda, del USNM de Washington, examinó la pequeña colección de estos crustáceos que 
recogió la mencionada expedición francesa y reconoce una subespecie y dos especies para el litoral de Argentina, asignándole posteriormente a estas últimas subgénero: Pterygosquilla armata armata $(\mathrm{H}$. Milne-Edwards), Heterosquilla (Heterosquilla) platensis (Berg) y Heterosquilla (Heterosquilla) polydactyla (von Martens). Asimismo, Christoffersen (1979) estudió los decápodos alfeidos de las campañas del «Calypso», describiendo algunas especies que se hallan en las costas de Argentina, particularmente Alpheus puapeba.

\section{La obra «Biologie de l'Amerique Australe»}

En la gran obra «Biologie de l'Amérique Australe» dirigida por Delamare (París) y Rapoport (Bahía Blanca) realizada con el auspicio del Centre National de la Recherche Scientifique de Francia y el Consejo Nacional de Investigaciones Científicas y Técnicas de la Argentina,en sus cuatro voluminosos tomos, figuran estudios sobre crustáceos de aguas continentales, costeras y del suelo. Rouch (1962) estudió los copépodos Harpacticoida de cuerpos de agua de la Provincia de Buenos Aires, de la Patagonia andina y de aguas marinas costeras. Delamare y Roland (1963) estudiaron crustáceos del suelo (sincáridos), Vandel (1963) isópodos terrestres de Tucumán y Andes Patagónicos, y Ringuelet (1968) trató la biogeografía de copépodos de agua dulce.

\section{Las primeras investigaciones sobre centolla y} otros eumalacostracos

Entre fines de 1958 y 1959 se realizó una campaña destinada a la búsqueda de concentraciones de centolla (Lithodes santolla) en el litoral patagónico, con el pesquero «Taiyo Maru 22», empleando redes enmalladoras (Angelescu, 1960). Por otra parte, los estudios sobre este crustáceo se iniciaron en Ushuaia (Canal Beagle) en 1973, con algunos muestreos. En 1974 se llevó a cabo una campaña de exploración pesquera con el BIP de la FAO, asociado al proyecto de Argentina, «Cruz del Sur», realizando mediciones de centollas y centollones y marcaciones de la primera especie (Fenucci et al., 1974). Un resumen de los conocimientos disponibles sobre la centolla en aquellos años fue realizado por Scelzo et al. (1974) y presentado en la reunión de la CARPAS (FAO) que se realizó en Montevideo. Posteriormente, mediante un convenio con el proyecto de la FAO, el ex-Instituto de Biología Marina y la Provincia de Tierra del Fuego, se llevó a cabo una serie de estudios de la centolla, cuyos resultados fueron expuestos en cuatro informes técnicos (Boschi et al., 1975-1976). Los trabajos sobre la biología pesquera de esta especie se interrumpieron, continuándose recién a partir de 1980, como se verá más adelante.

Una revisión de la familia Lithodidae (centollas y centollones) del océano Atlántico, fue realizada por Macpherson (1988), del Instituto de Ciencias del Mar de Barcelona. El trabajo, menciona 30 especies para esa área, varias descritas como nuevas. Además, el autor reivindica la primera designación específica de la centolla austral, de manera que el nombre Lithodes antarcticus, fue reemplazado por el de Lithodes santolla.

En cuanto a estudios faunísticos y como contribución del Instituto de Biología Marina de Mar del Plata, Boschi (1964) reunió todas las especies conocidas de Brachyura del litoral de la Provincia de Buenos Aires, lo que constituyó el primer aporte de conjunto para el mejor conocimiento de estos decápodos, y su distribución.

Escofet $(1970,1973)$ realiza interesantes aportes al conocimiento de los anfípodos del litoral bonaerense. En la revisión de las especies del género Pilumnoides (Xanthidae) Guinot y Macpherson (1987), tratan la especie argentina $P$. hassleri y consideran que en el material recibido de Argentina, puede existir una especie no descrita.

\section{La biogeografía}

En aspectos biogeográficos, Boschi (1966, 1976, 1979) en tres contribuciones sobre los decápodos marinos del litoral de Argentina, incluye todas las especies conocidas hasta esas fechas, actualiza la distribución de las mismas y las compara con otros organismos marinos de la región, reconociendo dos subregiones biogeográficas: la templada cálida y la templada fría (Provincias Magallánica y Argentina, respectivamente, según los malacólogos). Estos trabajos fueron complementados con un aporte relacionado exclusivamente con la distribución de los Anomura en el Atlántico Sudoccidental (Scelzo y Boschi, 1973).

\section{Los calianásidos, caprélidos y porcelánidos}

Biffar (1971) describe una especie nueva de Callianassidae para el litoral de Argentina, y Manning y Felder (1991) y Manning y Lemaitre (1993) clarifican la taxonomía de las especies americanas de este grupo. Las especies reconocidas para la Argentina son: Anacalliax argentinensis, Notiax brachyophthalma y Sergio mirim.

McCain (1965) en su estudio sobre caprélidos, examina una pequeña colección de anfípodos de Mar del Plata, identificando para el área a la especie cosmopolita Caprella equilibra Say. 
Bremec y Cazzaniga (1984) realizan un estudio comparativo entre las dos especies de porcelánidos mencionados para el litoral argentino: Pachycheles haigae y $P$. chubutensis, lo que amplía y complementa la primera contribución sobre el grupo efectuada por Boschi (1963).

\section{Un especialista argentino de amplio espectro}

A partir de 1966 Ramírez comienza sus pormenorizados trabajos sobre copépodos marinos, (Ramírez, 1966, 1969, 1970, 1971a, 1975, 1976, 1977, 1981b; Ramírez y Escofet, 1973; Ramírez et $a l .$, 1974). Por otra parte el mismo autor, con una aquilatada experiencia en organismos planctónicos, ha realizado estudios sobre eufáusidos (Ramírez, 1971b, 1973; Ramírez y Dato, 1983, 1989), cladóceros (Ramírez, 1981a; Ramírez y de Vreese, 1974), ostrácodos (Ramírez y Pérez, 1981a y 1981b), anfípodos hipéridos (Ramírez y Viñas, 1985). Asimismo, Ramírez (1967) estudió los ostrácodos de agua dulce de la Provincia de Buenos Aires, reconociendo 25 especies. Ultimamente, Moguilevsky y Whatley (1995) han tratado este complicado grupo.

\section{Los isópodos serólidos}

En una serie de trabajos, Bastida y Torti (1967a, 1967b, 1969, 1970) contribuyeron al estudio de los isópodos marinos, describiendo nuevas especies, ampliando su distribución y estudiando especialmente las especies de Serolidae de la expedición del «Calypso» a América del Sur. Se debe recordar que (Bastida, 1973) citó por primera vez a los cefalocáridos para el litoral argentino.

\section{Aportes del extranjero}

En un trabajo sobre los invertebrados bentónicos de las aguas marinas templadas de América del Sur, Semenov (1972) realiza una serie de interesantes consideraciones biogeográficas revisando la distribución de cerca de 300 especies, que incluye numerosos representantes de los crustáceos decápodos.

En la revisión de Ovalipes (Portunidae) Stepehnson y Rees (1968) separan en su distribución las especies $O$. punctatus y $O$. trimaculatus, dejando la primera para los mares de Japón y China, y la segunda para ambas costas de Sudamérica y sur del océano Indico.

Christoffersen (1988) estudiando los crangónidos, describe una especie nueva para el litoral de Argentina y aporta varias citas más.

Mazzocchi e Ianora (1991) estudiaron las asociaciones de copépodos del estrecho de Magallanes, recogidos en una campaña italiana . Más recientemente Guglielmo e Ianora (1995), estudian los copépodos del estrecho de Magallanes.

\section{La continuación de los estudios pesqueros}

De los primeros trabajos sobre crustáceos decápodos de interés pesquero, se puede mencionar, aparte del citado anteriormente para el langostino, el que trata sobre la dinámica poblacional del camarón Artemesia longinaris de las aguas costeras de Mar del Plata, realizado sobre la base de muestreos de 1965, 1966 y 1967, que significó el análisis de más de 30.000 especímenes, con interesantes conclusiones en cuanto a su reclutamiento, crecimiento y mortalidad (Boschi, 1969).

\section{Trabajos de conjunto que incluyen especies argentinas}

Williams (1974), un carcinólogo senior de USNM de Washington, en un trabajo sobre los cangrejos portúnidos del género Callinectes ubica a $C$. sapidus en la región rioplatense, como fuera señalado por Ringuelet (1963) para la costa Argentina, pero se han hallado excepcionalmente también algunos ejemplares en el litoral de la Provincia de Buenos Aires. Esta especie es la denominada «blue crab» de alto valor económico en EE.UU.

Holthuis, de Leiden, Holanda, uno de los especialistas en crustáceos más experimentados y reconocidos en el mundo, estudia una especie de langosta que había sido hallada en aguas del talud continental de la Argentina, en varios viajes y expediciones de barcos argentinos y extranjeros. Holthuis determinó a esta langosta de aguas profundas, como una nueva especie. Entre tanto Zarenkov y Semenov (1972) publicaron la nueva especie con material también del Atlántico Sur, recogido por barcos rusos, como Nephropides birsteini. Holthuis (1974), en su revisión de las langostas Nephropidea, asigna esta especie a un nuevo género y nueva subfamilia, denominándola Thymops birsteini, subfamilia Thymopinae. Por otra parte, este carcinólogo en una nueva edición de su obra «The Recent Genera of the Caridean and Stenopodidean Shrimps» toma las ilustraciones de trabajos producidos en la Argentina, para mencionar géneros con representantes en el Atlántico Sudoeste (Holthuis, 1993).

\section{Inventario de las especies de Decapoda del Mar Argentino}

Balech, renombrado fitoplanctólogo argentino, también realizó alguna incursión en crustáceos, mencionando por primera vez para el litoral de la pro- 
vincia de Buenos Aires a Scyllarides deceptor (Balech, 1962).

Mientras tanto, Torti y Boschi (1973) estudian las especies de camarones carideos del género Campylonotus, complementado con el trabajo de Boschi (1973) sobre tres especies de aguas profundas de camarones del mismo grupo.

Los cangrejos comensales que habían sido tratados muy parcialmente hasta ese momento, fueron estudiados por Fenucci (1975) quien registra ocho especies para las aguas marinas de Argentina, con la descripción de una nueva especie.

Otro importante trabajo sobre los Solenoceridae (Penaeoidea) americanos, lo realiza la carcinóloga cubana Pérez Farfante (1977), radicada desde hace varias décadas en EE.UU., y que trabaja en el U. S. National Museum of Natural History, reubica al langostino argentino en su antiguo género Pleoticus, en razón de que Burkenroad (1936) lo había incluido en el género Hymenopenaeus. Esta misma autora incluye las dos especies de peneidos de interés pesquero de la Argentina en una clave ilustrada de los Penaeoidea comerciales de las américas (Pérez Farfante, 1988).

Ultimamente, Boschi et al. (1992) prepararon un catálogo ilustrado de todos los crustáceos decápodos marinos de Argentina, reuniendo toda la información dispersa y actualizando su distribución.

\section{Los estudios en la Antártida}

Las investigaciones de los crustáceos de la Antártida, particularmente del krill (Euphausia superba) se vienen desarrollando desde hace varios años a través del Instituto Antártico Argentino. El biólogo responsable del proyecto es E. Marschoff, quien ha realizado numerosas campañas a la Antártida con relevantes resultados (Marschoff y Tomo, 1984).

\section{El Servicio de Hidrografía Naval Argentino}

Esta institución ha colaborado permanentemente en la coleccción de material biológico con sus buques hidrográficos. En las campañas participan los biólogos dedicados a estudios de la fauna y flora marinas, pertenecientes a museos y universidades, en los que figuran colecciones de crustáceos. En las campañas más recientes (1968-1971), se han obtenido valiosas muestras de zooplancton, que permitieron el estudio de los crustáceos .

\section{Los decápodos de agua dulce}

Los estudios sobre los cangrejos de agua dulce de la familia Trichodactylidae en la Argentina, tomaron impulso gracias a los trabajos de Lopretto (1976,
1980, 1981), quien siguió los primeros pasos dados sobre el tema por Ringuelet (1949). Rodríguez (1992), trata la mencionada familia a nivel continental, proponiendo importantes cambios genéricos y subgenéricos. Boschi (1981a) en la obra «Fauna de agua dulce de la República Argentina» trata las cuatro especies de camarones de agua dulce conocidos para la Argentina: tres palemónidos y un sergéstido.

\section{El desarrollo larval y embrionario de decápodos}

En el transcurso de los años sesenta comenzaron los trabajos sobre desarrollo larval de crustáceos decápodos marinos (Boschi y Scelzo, 1968; Boschi et al., 1967, 1968, 1969; Menú-Marque, 1970) y en los setenta se incian las experiencias de cultivos con las dos especies de peneidos de interés pesquero, langostino y camarón. En el Simposio de Montevideo de diciembre de 1974, organizado por la FAO sobre «La Acuicultura en América Latina», se presenta un trabajo referido al desarrollo larval y cultivo del camarón Artemesia longinaris, donde se exponen los primeros resultados obtenidos en la $\mathrm{Ar}$ gentina (Boschi y Scelzo, 1977). Esa línea de trabajo se extendió por varios años, con el estudio de las larvas y postlarvas de múltiples especies del litoral bonaerense. Un trabajo de interés ha sido el realizado sobre el langostino (Pleoticus muelleri), consistente en completar todo el desarrollo larval en laboratorio y lograr la producción masiva de postlarvas (Scelzo y Boschi, 1975). Posteriormente, Iorio et al. (1990) completan el estudio morfológico de las larvas y postlarvas, con material del plancton y de cultivo en el laboratorio y Mallo (1986) publica, como parte de su trabajo de tesis, el desarrollo larval del sergéstido Peisos petrunkevitchi en laboratorio

Los trabajos mencionados anteriormente fueron complementados con el estudio del desarrollo embrionario de $P$. muelleri en condiciones de laboratorio por Sarlo (1995), cuyo aporte es totalmente original en ese aspecto.

\section{La colaboración en el estudio del mar con nacio- nes amigas}

En el transcurso de 1978 y 1979 se realizaron en todo el Mar Epicontinental Argentino y su talud adyacente, numerosas campañas de investigación pesquera, mediante convenio entre el Gobierno Nacional (Instituto Nacional de Investigación y Desarrollo Pesquero (INIDEP), de Mar del Plata) y los gobiernos de Alemania y Japón, empleando naves 
de investigación de esos países: el nuevo BIP «Walther Herwig» de Alemania y el BIP «Shinkai-Maru» de Japón. De los resultados de esas campañas aparecieron dos trabajos. Uno se refiere a la distribución y abundancia de los crustáceos decápodos capturados en las campañas en la región explorada (Boschi et al., 1981) y otro de Ramírez (1981b) sobre copépodos, como parte del volumen editado por Angelescu sobre los resultados de la parte argentina de las campañas. Un tercer trabajo fue publicado en Japón, con el título «Decapod Crustacea off Patagonia», realizado por Takeda (1986), sin aportar nuevos datos de interés.

\section{Estudios complementarios realizados por especia- listas argentinos}

Pallares (1968) realizó excelentes contribuciones sobre los copépodos de la Ría de Deseado, asociándose con M. Hall para concretar aspectos más cuantitativos sobre el mismo grupo asociado a los bosques de algas Macrocystis (Pallares y Hall, 1974). Delgado de Layño y Pallares (1981) estudiaron el contenido de lípidos en el copépodo Tigriopus californicus.

Montú (1977) realizó un estudio sobre la distribución estacional de los eufáusidos del mar patagónico. Por otra parte, Lopretto (1982, 1983a, 1983b) contribuyó con varios trabajos sobre la bioecología del anfípodo de agua dulce Hyalella pampeana, que fuera descripto por Cavalieri (1968).

Carreto et al. (1981), empleando muestras de las campañas de los buques BIP «Walther Herwig» y «Shinkai-Maru», realizaron estudios de la distribución y variación estacional de la biomasa de zooplancton (integrado básicamente por macro y microcrustáceos) en todo el Mar Epicontinental Argentino y talud adyacente.

Mallo y Boschi (1982) estudian el ciclo vital del camarón Peisos petrunkevitchi de Mar del Plata, un pequeño camarón sergéstido que resultó ser una especie clave en las tramas tróficas costeras bonaerenses. Este trabajo amplía los estudios previos de Boschi (1965) y de Boschi y Scelzo (1969).

Ciechomski y Sánchez (1983) estudiaron la relación entre el ictioplancton y la abundancia de la biomasa de zooplancton (especialmente copépodos) en aguas de la plataforma de Argentina.

Roux et al. (1995) estudiando las comunidades bentónicas de los fondos de pesca del langostino en el golfo San Jorge, realizan una exhaustiva enumeración de los crustáceos decápodos hallados en los arrastres con los barcos de investigación, destinado al monitoreo del mencionado peneido. Roux y Bremec (en prensa), estudian la fauna bentónica obtenida en tres secciones realizadas frente al Río de la Plata, Mar del Plata y Península Valdés, enumerando los decápodos hallados. Las mismas autoras realizan un estudio de las comunidades bentónicas asociadas a bancos de mejillones donde hallan numerosos crustáceos (Bremec y Roux, en prensa).

\section{El «Atlas del zooplancton»}

Esta obra magnífica, concebida, dirigida y editada por D. Boltovskoy, como una publicación especial del INIDEP en 1981, trata del zooplancton, métodos de estudio y oceanografía del Atlántico Sudoccidental. Fue preparada por un grupo de especialistas nacionales e internacionales, e incluyó varios capítulos sobre crustáceos, tales como cladóceros por Ramírez (1981), ostrácodos por Angel (1981), copépodos por Bjornberg (1981), eufausiáceos por Antezana y Brinton (1981) y larvas de crustáceos decápodos por Boschi (1981), lo cual constituyó un importante avance en el conocimiento de estos invertebrados marinos de esta región.

\section{El auge del estudio de los crustáceos}

A partir de los años ochenta, el número de biólogos dedicados al estudio de los crustáceos , creció considerablemente. Se destacan los trabajos de Cohen (1981, 1982, 1983, 1985, 1987, 1991a, 1991b, 1992, 1993, 1995) y de Cesar (1985, 1987a, 1987b, 1989a y 1989b), sobre Anostraca. Los Conchostraca y Notostraca fueron revisados por Lopretto (1995a, 1995b). De estos últimos se reconocen Lepidurus apus patagonicus de la región templada fría de Argentina y Triops longicaudatus longicaudatus, para las provincias de Córdoba, La Pampa, y la región oeste entre Río Negro y La Rioja. Sobre los Conchostraca se mencionan especies de los géneros Cyclestheria, Leptestheria y Limnadia, para la región central norte de Argentina.

Roccatagliata $(1990,1993)$ investiga los Cumacea, con interesantes y novedosos hallazgos mediante el estudio de muestras de distintas regiones oceánicas y de aguas dulces, continuando la primera mención del grupo realizada por Bachmann y Moguilevsky (1973) para el Río de la Plata. Roccatagliata, en pocos años, se ha constituido, en un especialista de nivel mundial.

Los estudios sobre crustáceos decápodos fósiles en los recientes años han sido tratados por Aguirre-Urreta y Ramos (1981) y Aguirre-Urreta (1982), se refieren a material de Astacidea del 
Cretácico Inferior de la cuenca austral de la provincia de Santa Cruz. Los Brachyura Geryonidae del terciario de la Patagonia y Tierra del Fuego (Argentina) fueron revisados por Aguirre-Urreta (1987), describiendo especies de Coeloma y Lebucarcinus, haciendo referencia al género Geryon que contiene una especie actual, que fuera mencionada para el Atlántico Sudoccidental por Scelzo y Valentini (1974). La misma autora estudia decápodos del Cretácico de Argentina continental y de la Antártida (Aguirre-Urreta, 1989).

Aguirre-Urreta y colaboradores continúan con estudios sobre decápodos fósiles. Sobre langostas (Aguirre-Urreta et al., 1990; Aguirre-Urreta et al., 1991; Aguirre-Urreta, 1992) cangrejos ermitaños (Aguirre-Urreta y Olivero, 1992).

Recientemente, Manning y Holthuis (1989) realizan una revisión total de las especies vivientes de la familia Geryonidae ubicando al taxón del Atlántico Sudoccidental (Uruguay y Argentina), en el nuevo género Chaceon con la denominación específica Chaceon notialis. La familia necesitaba un estudio de conjunto, debido a que las especies de distintas regiones del océano eran designadas en su mayoría como Geryon affinis o G. quinquedens.

\section{Las tesis doctorales sobre crustáceos}

Varias tesis doctorales se presentaron en la Facultad de Ciencias Exactas y Naturales de la Universidad de Buenos Aires sobre temas relacionados con los crustáceos. Entre ellas se pueden mencionar la de Vinuesa (1982), sobre biología reproductiva y desarrollo embrionario de la centolla del Canal Beagle (1982), y la de Alonso (1984) sobre anfípodos gammáridos litorales. Bacardit (1986) analizó las larvas de crustáceos decápodos del plancton del mar patagónico. Spivak (1987) sobre la muda y el crecimiento de Cyrtograpsus angulatus. Petriella (1987) sobre el ciclo de muda del camarón Artemesia longinaris: descripción, crecimiento y relación con el colesterol. Roccatagliata (1990) en sistemática y biología de cumáceos. Cohen (1991) en anostracos de la familia Branchinectidae. Dimant (1991) sobre comportamiento exploratorio del cangrejo Chasmagnathus granulata (sobre comportamiento figuran varias tesis más). Lovrich (1991) en reproducción y crecimiento del centollón Paralomis granulosa del Canal Beagle. Comoglio (1994) en la nutrición de los crustáceos decápodos en el Canal Beagle, con especial énfasis en la centolla y el centollón, y la función trófica de los mismos en el ecosistema. Estas menciones no incluyen segura- mente la totalidad de las tesis presentadas que se relacionan con los crustáceos. Casi todos estos jóvenes biólogos hoy día trabajan sobre temas afines a las respectivas tesis.

En la Universidad Nacional del Sur, Cervellini (1987) estudió las larvas y postlarvas de crustáceos de la Bahía Blanca (Provincia de Buenos Aires) y Sabatini (1987) la dinámica poblacional del cópepodo Acartia tonsa y su producción en Bahía Blanca.

Moreno (1977) de la Universidad Nacional de La Plata, analizó la composición de lípidos y biosíntesis de dos organismos del zooplancton, uno de ellos el copépodo Paracalanus parvus. Mallo (1984) hizo su tesis sobre desarrollo larval y cultivo del sergéstido Peisos petrunkevitchi, muy abundante en las aguas costeras marplatenses y que sirve como alimento a un variado número de peces carcinófagos.

En la Universidad Nacional de Mar del Plata, Jeckel (1990) hizo su tesis sobre la composición bioquímica, lipídica y de ácidos grasos del langostino ( $P$. muelleri) en relación con factores ecológicos. Este investigador ha seguido trabajando y publicando en esa línea (Jeckel et al., 1989, y otros). Marcovecchio (1988) preparó su tesis sobre contaminación con metales en varios organismos, incluyendo el langostino y camarón en dos áreas estuariales de Argentina. Fernández (1994) efectuó su tesis sobre biomasa de copépodos, en especial calanoideos recogidos en campañas de investigación pesquera del INIDEP.

\section{Los problemas de la contaminación}

Los estudios sobre contaminación del medio han sido desarrollados por varios investigadores. Particularmente Marcovecchio (1994) estudió la presencia de metales traza, mercurio, cadmio y zinc en el langostino $P$. muelleri y camarón A. longinaris en Bahía Blanca, considerándolos como bioindicadores.

\section{Los cultivos de peneidos}

Los estudios sobre cultivos de camarones comerciales peneidos han tenido un desarrollo continuo en Mar del Plata. Luego de los primeros trabajos en el ex-Instituto de Biología Marina, de 1972 a 1974, Fenucci al regresar de su especialización en Texas, EE.UU., inició trabajos sobre fisiología de la nutrición de crustáceos, y constituyó un grupo de trabajo en aspectos de alimentación y engorde de peneidos, con la publicación de importantes resul- 
tados. El centro de cultivos de camarones está en la Estación NAGERA, dependiente de la Universidad Nacional de Mar del Plata. Se puede mencionar algunos trabajos del equipo: Petriella et al. (1984) sobre la influencia de dietas de ácidos grasos y colesterol sobre el crecimiento y supervivencia de Artemesia longinaris; Fenucci et al. (1990) sobre la factibilidad de cría del langostino $P$. muelleri; Haran (en prensa) en relación con la acción de los ácidos grasos sobre el crecimiento del langostino; Mallo et al. (en prensa) sobre la cría masiva de larvas y postlarvas de langostino, y Mallo (en prensa) sobre la influencia de la temperatura, salinidad y contaminantes en dos especies de peneidos.

Petriella ha trabajado dentro del grupo de Fenucci pero con cierta independencia. Realizó contribuciones sobre fisiología de la muda y crecimiento experimental en peneidos (Petriella, 1984, 1986, 1987; Petriella y Bridi, 1992; Petriella et al., 1984; Fenucci et al., 1981 y otros) y varios trabajos realizados en colaboración con Díaz (Petriella y Díaz, 1987; Díaz y Petriella, 1988).

Dentro del grupo de estudios de biología y cultivos de crustáceos decápodos, se destaca asimismo la labor de Marcelo A. Scelzo, quien integró el equipo desde sus comienzos en el ex-Instituto de Biología Marina de Mar del Plata (Boschi y Scelzo, 1968, 1973, 1977; Scelzo y Boschi, 1975, Scelzo, 1973; Iorio et al., 1990 y otros). Merece ser mencionada su tarea en la formación de estudiantes y graduados en las disciplinas carcinológicas en Argentina (Universidad Nacional de Mar del Plata) y Venezuela.

\section{La carcinología de campo y de laboratorio}

Spivak comenzó estudios sobre incrustaciones e isópodos marinos. Se estableció en la Universidad Nacional de Mar del Plata y se dedica a estudios poblacionales y biología de cangrejos estuariales, creando un interesante grupo de estudio de grápsidos del mediolitoral (Spivak y Politis, 1989; Spivak et al., 1991; Spivak, 1988; Spivak, 1990 y otros).

Tablado y López (1995) han realizado observaciones sobre la incidencia del pinotérido (Tumidotheres maculatus) comensal del mejillón (Mytilus edulis), que lo consideran como un caso de parasitismo.

\section{La evaluación de los recursos}

Con la creación del INIDEP en Mar del Plata, dependiente de la Secretaría de Agricultura, Pesca y Alimentación, se intensificaron los estudios de crustáceos decápodos de interés pesquero, tales como centolla (L.santolla), langostino (P. muelleri), centollón ( $P$. granulosa), y camarón (A. longinaris). La realización de numerosas campañas con los barcos de investigación del INIDEP «Cap. Oca Balda», «Dr. Eduardo L. Holmberg» y «Cap. Cánepa» en todo el Mar Epicontinental y talud adyacente, permitió disponer de excelente material de estudio sobre la presencia, distribución y abundancia de la mayoría de las especies que fueron capturadas con las artes de pesca, rastras y redes de plancton empleadas por los BIP de investigación. De estas campañas y viajes a la región sur (Tierra del Fuego y Canal Beagle) han surgido varios trabajos: Boschi et al., (1984) y Bertuche et al., (1990).

También se intensificaron los estudios sobre la evaluación del recurso langostino en el litoral patagónico, debido al importante incremento de las capturas, que en algunos años, a partir de 1981, llegaron a casi 25.000 ton. El INIDEP destinó varias campañas anuales a monitorear el recurso y asesorar a las administraciones pesqueras nacionales, provinciales, y a la industria. Sobre la base de estos primeros trabajos, que significó la interpretación de 17 campañas con los BIPs. del INIDEP, entre 1982 y 1988 han surgido, además de los resultados biológicos, pautas para el manejo de este recurso (Boschi, 1989).

Por otra parte Macchi et al. (1992) estudiaron aspectos del desove y fecundidad del langostino. Asimismo, Fischbach (1993) realizó investigaciones sobre la dinámicas de sus larvas y postlarvas en el litoral bonaerense. Bertuche et al. (1987) propusieron un modelo bioeconómico para el manejo del recurso langostino patagónico sobre la base de considerar como una unidad el recurso de esa región, pero no ha tenido aplicación por parte de la administración, ni de la industria dedicada a la extracción de este crustáceo.

El monitoreo del recurso langostino continúa actualmente, por parte del INIDEP, con la realización de varias campañas durante el año, de manera tal que se dispone de datos permanentes sobre su ciclo vital y fluctuaciones de su abundancia en el litoral patagónico.

\section{EI CADIC de Ushuaia}

Otro laboratorio de estudios carcinológicos es el Centro Austral de Investigaciones Científicas (CADIC), dependiente del Consejo Nacional de Investigaciones Científicas y Técnicas (CONICET), ubicado en la ciudad de Ushuaia, Tierra del Fuego, que desde hace más de una década ha mantenido estas líneas de investigación conducidas por J.H. 
Vinuesa, en colaboración con un grupo de jóvenes biólogos, radicados en esas alejadas tierras australes, donde se han efectuado estudios sobre la biología, desarrollo gonadal, crecimiento, alimentación y distribución de crustáceos decápodos del Canal Beagle, particularmente de la centolla y centollón (Vinuesa, 1984; 1985; Vinuesa et al., 1989; Lovrich y Vinuesa, 1993; Comoglio et al., 1990; Lovrich, en prensa; Lovrich y Vinuesa 1995 y en prensa), entre otros.

\section{La fisiología del comportamiento y estudios experimentales}

H. Maldonado, de la Facultad de Ciencias Exactas y Naturales de la Universidad de Buenos Aires, realiza estudios de fisiología del comportamiento con el cangrejo estuarino Chasmagnathus granulata constituyendo un grupo de trabajo de reconocimiento internacional, con la producción de trabajos publicados en las mejores revistas de la especialidad.

Rodríguez, con un grupo de colaboradores realiza investigaciones sobre la acción de pesticidas (parathion) sobre el cangrejo de estuario $C$. granulata (Rodríguez y Amin, 1991; Rodríguez y Lombardo, 1991; Rodríguez y Monserrat, 1991; Monserrat et al., 1991; Rodríguez et al., 1992; Rodríguez y Pisanó, 1993).

\section{Las colecciones carcinológicas}

Las principales colecciones carcinológicas en la República Argentina están localizadas en los Museos de Ciencias Naturales. En general son modestas y no se observa que sus colecciones aumenten rápidamente. Por lo general dependen de la presencia de algún especialista interesado que ingrese al museo y le dé un nuevo impulso a las colecciones. Se pueden mencionar entre las más importantes las del Museo Argentino de Ciencias Naturales «Bernardino Rivadavia» de Buenos Aires y la del Museo de Ciencias Naturales de La Plata. En el Departamento de Ciencias Biológicas de la Facultad de Ciencias Exactas y Naturales de la Universidad de Buenos Aires, existen colecciones de algunos crustáceos en razón de la presencia de varios especialistas. También existe una colección más reciente en el INIDEP, en Mar del Plata, obtenida de las múltiples campañas de investigación.

\section{La revista «Crustaceana» publicada en}

\section{Leiden}

Esta revista ha tenido mucha influencia a nivel mundial, por ser una publicación especializada sobre crustáceos, con excelentes trabajos y de amplia distribución internacional. Se ha mantenido desde su primer número, aparecida en enero de 1960, con continuidad y prolija impresión. Está editada por E.J. Brill, Leiden, Holanda. Algunos trabajos de carcinólogos argentinos han salido publicados en Crustaceana.

\section{The Crustacean Society de EE.UU.}

La Crustacean Society de EE.UU. fue creada en 1979. organizada por varios carcinólogos americanos (Manning, 1990). La publicación de su prestigiosa revista «Journal of Crustacean Biology» se inició en febrero de 1981 y aparece cuatro veces al año. La referida Sociedad ha tenido repercusión en toda América y varios especialistas argentinos son miembros de la misma. Esta sociedad organiza reuniones periódicamente y en varias de ellas ha participado un importante número de carcinólogos latinoamericanos.

\section{Asociación Argentina de Carcinología}

Se inició en el transcurso de las VII Jornadas Argentina de Zoología, en Mar del Plata, en octubre de 1984. Edita un boletín de aparición irregular. Mantiene suscripciones a varias revistas especializadas y adquiere libros sobre crustáceos. Cumple la importante misión de mantener un vínculo de comunicación entre los especialistas argentinos. Un grupo de carcinólogos del Departamento de Biología de la Facultad de Ciencias Exactas y Naturales de la Universidad de Buenos Aires, la mantiene en actividad. Se puede mencionar a Roccatagliata y a Cohen, entre otros, por lo cual mucho le debemos agradecer. Una última lista de carcinológos preparada por la Asociación (junio 1995), indica la existencia de 74 especialistas, incluyendo algunos colegas uruguayos.

\section{Asociación Latinoamericana de Carcinología}

En el transcurso del VI Congreso Latinoamericano de Ciencias del Mar, realizado en Mar del Plata, Argentina, en octubre de 1995, por iniciativa de un grupo de biólogos dedicados al estudio de los crustáceos se creó la Asociación Latinoamericana de Carcinología, con el fin de promover los estudios sobre estos invertebrados en Latinoamérica y estimular el intercambio entre especialistas. El acta fue suscrita por representantes de Brasil, Cuba, Venezuela, Chile, México, Perú y Argentina. Es de esperar que la Asociación sirva para impulsar el interés por la carcinología en nuestro continente. 


\section{CONSIDERACIONES FINALES}

Esta breve reseña de la carcinología en la Argentina ha resultado ser más compleja de lo que algún carcinólogo podía imaginar. No se hallarán en las páginas anteriores muchas figuras ilustres ni una larga tradición de acontecimientos ni hechos históricos demasiado sobresalientes, como se puede observar en el libro editado por F. Truesdale (1993) History of Carcinology. Lo que resulta sorprendente y auspicioso es el número importante de trabajos de autores argentinos que han aparecido en las últimas dos décadas estudiando a los crustáceos. Cuando Ringuelet (1961) en las Sesiones Cientifícas de Zoología, realizadas en Tucumán, en noviembre de 1960, da un «Panorama Zoológico Argentino», referido a los invertebrados excepto insectos, resume en una página y media lo concerniente a la carcinología argentina, comenzando con Berg y finalizando con algunos pocos trabajos realizados en 1959.

Contrariamente, en esta reseña se hallarán muchas omisiones, porque es imposible reunir y comentar, en tan breve plazo, tantos trabajos de autores argentinos aparecidos en los últimos años en revistas nacionales e internacionales, por lo cual muchos especialistas se verán mencionados con pocas contribuciones. Cuando mi amigo y colega I. Wehrtmann, de la Universidad Austral de Chile, me propuso presentar este trabajo en el curso internacional de postgrado, en Iquique, en marzo de 1996, no pensé que ello me iba a exigir más tiempo del que pude dedicarle, por lo que pido mil discupas por las múltiples imperfecciones. No obstante puedo expresar que disfruté de su elaboración y por momentos me absorbió totalmente su redacción. Ha sido de suma utilidad disponer del tratado Ecosistemas de Aguas Continentales, editado por Lopretto y Tell (1995), de permanente consulta referida a los trabajos sobre crustáceos de aguas continentales de la Argentina.

Soy de opinión que queda la posibilidad de perfeccionar la compaginación y redacción de este trabajo, y agregarle las numerosas contribuciones que no pudieron ser incorporadas al texto, así como la mención de todos los biólogos que en algún momento de su vida científica estudiaron los crustáceos de la Argentina. No obstante ello, es posible pensar que se debía comenzar en algún momento un trabajo que no había sido hecho, como en este caso, aunque se realizó en circunstancias poco previstas y con un tiempo muy acotado. Sobre esa base es más simple su mejoramiento y ampliación. Deseo agradecer las observaciones de dos árbitros anónimos que han contribuido a mejorar el texto.

\section{REFERENCIAS BIBLIOGRAFICAS}

Aguirre-Urreta, M.B. 1982. Crustáceos decápodos barremianos de la región del Tucu-Tucu, Prov.de Santa Cruz Ameghiniana, 19(3-4): 303-317.

Aguirre-Urreta, M.B. 1987. La familia Geryonidae (Crustacea: Brachyura) en el Terciario de Patagonia y Tierra del Fuego, Argentina. 4 Congr. Lat. de Paleontología, Bolivia, 1: 459-484.

Aguirre-Urreta, M.B. 1989. The Cretaceous Decapod Crustacea of Argentina and the Antarctic Peninsula. Paleontology, 32(3): 499-552.

Aguirre-Urreta, M.B. 1992. Tertiary freshwater Decapoda (Crustacea: Parastacidae) from the Ñirihuau bassin, Patagonia, Argentina. J. Paleont., 66(5): 817-825.

Aguirre-Urreta, M.B., L.A. Buatois, G.CH.B. Chernoglasov y F.A. Medina. 1990. First Polychelidae (Crustacea: Palinura) from de Jurassic of Antarctica. Antarctic Science, 2(2): 157-162.

Aguirre-Urreta, M.B., E.B. Olivero y F.A.Medina. 1991. A redescription of a Maastrichtian lobster Hoploparia antarctica Wilckens,1907 (Crustacea: Decapoda), from Chubut, Argentina. J. Paleont., 65(5): 795-800.

Aguirre-Urreta, M.B. y E.B. Olivero. 1992. A Cretaceous hermit crab from Antarctica: predatory activities and bryozoan symbiosis. Antarctic Science, 4(2): 207-214.

Aguirre-Urreta, M.B. y V.A. Ramos. 1981. Crutáceos decápodos del Cretácico inferior de la cuenca austral, Prov. de Santa Cruz, Argentina. Com. Sud. Jur. y Cret. Cuenc. Sediment. del Jurásico y Cret. de Am. del Sur, 2: 599-623.

Alonso, G.M. 1984. Anfípodos gammarideos litorales del mar austral argentino (Crustacea, Amphipoda, Gammaridea). Tesis doctoral, Universidad de Buenos Aires, 200 pp.

Andriyashev, A.P. y P.V. Ushavok. 1970. Biological reports of the Soviet Antarctic Expedition (1955-1958). (Translated from russian) 4: 1-292. 
Angel, M.V. 1981. Ostracoda. In: D. Boltovskoy. (ed.), Atlas del zooplancton del Atlántico Sudoccidental y métodos de trabajo con el zooplancton marino. INIDEP, Publicación especial, 543-585 pp.

Angelescu, V. 1960. Operación centolla en el Atlántico Sur. Serv. de Hidrogr. Naval, H1013: $1-71$.

Angelescu, V. y E.E. Boschi. 1959. Estudio biológico pesquero del langostino de Mar del Plata. Serv. Hidrogr. Naval, H1017: 1-135.

Antezana, T. y E. Brinton. 1981. Euphausiacea. In: D. Boltovskoy (ed.), Atlas del zooplancton del Atlántico Sudoccidental y métodos de trabajo con el zooplancton marino. INIDEP, Publicación especial, 681-698.

Bacardit, R. 1986. Larvas de crustáceos Decápoda del mar patagónico, incluyendo el talud continental, adyacencias a la Tierra del Fuego e Islas Malvinas. Aspectos morfológicos, ecológicos y filogenéticos. Tesis doctoral, Universidad de Buenos Aires, 297 pp.

Bacescu, M.C. 1962. Contribution à la connaissance du genre Makrokylindrus Stebbing (Crustacea, Cumacea). Vema Research Series, 1: 207-223.

Bachmann, A.O. y A.O. Moguilevsky. 1973. Sobre la presencia de crustáceos cumáceos Nannastacidae en la ribera argentina del Río de la Plata. Physis, Buenos Aires, A32(84): 219-220.

Balech, E. 1962. Nótulas de la Estación Hidrobiológica de Puerto Quequén. Rev. Mus. Arg. Cienc. Nat., Zool., 7(6): 81-87.

Barnard, J.L. 1962. South Atlantic abyssal amphipods. Vema Res. Ser., 1: 11-78.

Bastida, R. 1973. Sobre el hallazgo de Crustacea Cephalocarida frente a las costas argentino-uruguayas. Physis, Buenos Aires, A32(84): 220.

Bastida, R. y M.R. Torti. 1967a. Nuevas consideraciones sobre Serolis polaris. Richardson 1911. Neotropica, La Plata, 13(40): 31-39.

Bastida, R. y M.R. Torti. 1967b. Una nueva especie de Isopoda Serolidae para las costas de la Provincia de Buenos Aires (Argentina). Bull. Mus. Nat. d'Hist. Nat., 2a.Ser., 39(3): 573-582.

Bastida, R. y M.R. Torti. 1969.Crustáceos isópodos: Serolidae. Résultats Scient. Camp.de la Calypso, 9 Ann. Inst. Océan., Paris, 47: 61-105.
Bastida, R. y M.R. Torti. 1970. Chaetilia argenti$n a$ sp. nov., un nuevo aporte al conocimiento de la isopodofauna sudamericana. Bull. Mus. Nat. d'Hist.Natur., 2a Ser., 42(2): 392-400.

Bate, C.S. 1988. Report on the Crustacea Macrura dredged by H.S.M. Challenger during the years 1873-1876 (Rep. Sci. Res. Voyage Challenger, 1873-76. Zool.), Thomson C.W. Murray (ed.). J. London, 24, I - XC, 942 pp.

Battistoni, P.A. 1995. Crustacea Copepoda. Ecosistemas de aguas continentales, Metodologías para su estudio (E.C. Lopretto y Tell, G. Directores). Ediciones Sur, La Plata, 953-971 pp.

Berg, C. 1900. Datos sobre algunos crustáceos nuevos para la fauna argentina. Com. Mus. Nac. Buenos Aires, 1(7): 223-235.

Berg, C. 1901. Sobre el langostino y el camarón, dos crustáceos macruros de aguas argentinas y uruguayas. Contrib. Mus. Nacional, Buenos Aires, 1(2): 37-39

Bertuche, D.A., J.G. Wyngard, H. Hack, H. Abal y D.R. Hernández. 1987. Bioeconomic model of the argentine shrimp (Pleoticus muelleri) fishery of the San Jorge Gulf, Argentina. Proc. Intern. Conf. Fish. Univ. Quebec, Canada, pp. 395-419.

Bertuche, D.A., J. Wyngaard, C. Fischbach y E.E. Boschi. 1990. Population structural variation of the southern king crab Lithodes santolla of the Beagle Channel, Argentina, from 1975 to 1989. Proc. Int. Symp. King and Tanner crab, Nov.1989, Alaska, Anchorage, pp. 411-426.

Biffar, T.A. 1971. New species of Callianassa (Decapoda: Thalassinidae) from West Atlantic. Crustaceana, 21(3): 225-236.

Biraben, M. 1917. Nota sobre dos cladóceros nuevos de la República Argentina. Physis, Buenos Aires, 3(14): 262-266.

Biraben, M. 1918. Sobre algunos cladóceros de la República Argentina. Rev. Mus. La Plata, 24:82-126.

Biraben, M. 1939. Los cladóceros de la familia Chydoridae. Physis, Buenos Aires, 17(46): 651-671.

Biraben, M. 1946. Sobre dos nuevas especies de Branchinecta de la Patagonia (Phyllopoda). Notas Mus., La Plata, 11(94): 129-139. 
Biraben, M. 1951. Nuevo género de Phyllopoda Anostraca (Crustacea). Physis, Buenos Aires, 20(58): 324-329.

Biraben, M. 1954. Dos nuevas especies de Daphnia de la Argentina. Physis, Buenos Aires, 20(59):414-419.

Biraben, M. 1957. Sobre la distribución de Artemia salina en la Argentina. Neotropica, La Plata, 1(2): 31-32.

Bjornberg, T.K.S. 1981. Copepoda. In: D. Boltovskoy. (ed.), Atlas del zooplancton del Atlántico Sudoccidental y métodos de trabajo con el zooplancton marino. INIDEP, Publicación especial, 587-679 pp.

Boschi, E.E. 1963. Sobre dos especies de Pachycheles de la Argentina (Crustacea, Anomura). Neotropica, La Plata, 9(28): 31-37.

Boschi, E.E. 1964. Los crustáceos decápodos Brachyura del litoral bonaerense. Bol. Inst. Biol. Marina, Mar del Plata, 6: 1-99.

Boschi, E.E. 1965. Un interesante sergéstido nuevo para las aguas marinas de la Argentina (Crustacea: Decapoda), Physis, Buenos Aires, A25(69): 93-94.

Boschi, E.E. 1966. Preliminary note on the geographic distribution of the decapod crustaceans of the marine waters of Argentina (South West Atlantic Ocean). Symposium Proc. Crustacea. India, Part 1: 449-456.

Boschi, E.E. 1969. Estudio biológico pesquero del camarón Artemesia longinaris Bate de Mar del Plata. Bol. Inst. Biol. Marina, Mar del Plata, 18: $1-47$.

Boschi, E.E. 1973. Aportes al conocimiento de tres especies de camarones de aguas profundas del Atlántico Sur (Crustacea: Caridea). Physis, Buenos Aires, A32(85): 233-244 pp.

Boschi, E.E. 1976. Nuevos aportes al conocimiento de la distribución geográfica de los crustáceos decápodos del mar argentino. Physis, Buenos Aires, A35(90): 59-68.

Boschi, E.E. 1979. Geographic distribution of Argentinian marine decapod crustaceans. Bull. Biol. Soc. Wash., 3: 134-143.

Boschi, E.E. 1981. Larvas de Crustacea Decapoda. In: D. Boltovskoy (ed.), Atlas del zooplancton del Atlántico Sudoccidental y métodos de trabajo con el zooplancton marino. INIDEP, Publicación especial, 699-758.
Boschi, E.E. 1981a. Decapoda Natantia. Fauna de agua dulce de la República. Argentina, FECIC, 26: $1-61$.

Boschi, E.E. 1989. Biología pesquera del langostino del litoral patagónico de Argentina. INIDEP, Mar del Plata, Contrib., 646: 1-71.

Boschi, E.E. y V. Angelescu. 1962. Descripción de la morfología externa e interna del langostino con algunas aplicaciones de índole taxonómica y biológica. Bol. Inst. Biolog. Marina, Mar del Plata, 1: 1-73.

Boschi, E.E. y M.A. Scelzo. 1968. Larval development of the spyder crab Libinia spinosa in the laboratory. Crustaceana, Suppl., 2: 170180.

Boschi, E.E. y M.A. Scelzo. 1969. Nuevas campañas exploratorias camaroneras en el litoral argentino 1967-1968, con referencias al plancton de la región. Proyec. Des. Pesquero FAO-Argentina, Inf. Técnico, 16: 1-31.

Boschi, E.E. y M.A. Scelzo. 1973. Aportes al conocimiento de la distribución de los crustáceos Anomura del Atlántico Sudoccidental. V Cong.Lat. Zool., 1: 204-216.

Boschi, E.E. y M.A. Scelzo. 1977. Desarrollo larval y cultivo del camarón comercial de Argentina Artemesia longinaris Bate (Crustacea, Decapoda, Penaeidae). FA0 Inf. de Pesca, 159(1):287-327.

Boschi, E.E., D.A. Bertuche y J.G. Wyngaard. 1984. Estudio biológico pesquero de la centolla (Lithodes antarcticus) del Canal Beagle, Tierra del Fuego, Argentina. Contrib. INIDEP, Mar del Plata, 441: 1-71.

Boschi, E.E., C.E. Fischbach y M.I. Iorio. 1992. Catálogo ilustrado de los crustáceos estomatópodos y decápodos marinos de Argentina. Frente Marítimo, 10A: 7-94.

Boschi, E.E., B. Goldstein y M.A. Scelzo. 1968. Metamorfosis del crustáceo Blepharipoda doelloi Schmitt, de las aguas de la Prov. de Buenos Aires. Physis, Buenos Aires, 27(75):291-311.

Boschi, E.E., M.I. Iorio y K. Fischbach. 1981. Distribución y abundancia de los crustáceos decápodos capturados en las campañas de los B/I Walther Herwig y Shinkai Maru en el Mar Argentino, 1978-1979. Contrib. INIDEP, Mar del Plata, 383:233-253. 
Boschi, E.E., M.A. Scelzo y B. Goldstein. 1967. Desarrollo larval de dos especies de crustáceos Decápodos en el laboratorio Pachycheles haigae Rodrigues Da Costa (Porcellanidae) y Chasmagnathus granulata Dana (Grapsidae). Bol. Inst. Biol. Marina, Mar del Plata, 12: 1-46.

Boschi, E.E., M.A. Scelzo y B. Goldstein. 1969. Desarrollo larval del cangrejo Halicarcinus planatus (Fabricius) en el laboratorio (Crustacea, Decapoda, Hymenosomatidae), con observaciones sobre la distribución de la especie. Bull. Mar. Sci., Miami, 19(1): 225-242.

Boschi, E.E., M.A. Scelzo y R. Pérez. 1975-1976. Informes de trabajo sobre el convenio entre el Pro yecto de Desarrollo Pesquero (FAO), el Territorio Nacional de Tierra del Fuego, Antártida e Islas del Atlántico Sur y el Instituto de Biología Marina de Mar del Plata, relativo al estudio biológico pesquero de la centolla. Instituto de Biología Marina, Mar del Plata, 1: 15 pp., 2: 11 pp., 3: 7 pp. y 4: $6 \mathrm{pp}$.

Brehm, V. 1954. Sobre los copépodos hallados por el Prof. Birabén en la Argentina. Neotrópica, La Plata, 1(3): 37-42.

Bremec, C.S. y N.J. Cazzaniga. 1984 Consideraciones sobre Pachycheles haigae Rodrigues de Costa, 1960 y P. chubutensis Boschi, 1963 en Monte Hermoso (República Argentina) (Crustacea, Anomura, Porcellanidae). Iheringia, Sér. Zool., Porto Alegre, 64: 149-162.

Bremec, C.S. y A. Roux (en prensa). Resultados del análisis de una campaña de investigación pesquera sobre comunidades bentónicas asociadas a bancos de mejillones (Mytilus edulis platensis D'orb.). Rev. Invest. Des. Pesquero, Mar del Plata.

Brian, A. 1925. Di alcuni copepodi d'acqua dolce dell'Argentina, raccolti dal Prof. F. Silvestri. Mem. Soc. Ent. Ital., 4(2): 177-200.

Bruch, C. 1916. Dos curiosos crustáceos de San Luis. Physis, Buenos Aires, 2(12): 462-464.

Burkenroad, M.D. 1936. The Aristaeinae, Solenocerinae, and pelagic Penaeinae on the Bingham oceanographic collection. Bull. Bingham Oceanogr. Coll., Yale Univ., New Haven, 5 (Art.2): 1-151.

Carreto, J.I., F.C. Ramírez y C. Dato. 1981. Zooplancton y producción secundaria. Parte II. Distribución y variación estacional de la biomasa zooplanctónica. Contribución INIDEP, Mar del Plata, 383: 213-232.
Cavalieri, F. 1968. Hyalella pampeana sp. nov. una nueva especie de anfípodo de agua dulce (Gammaridea: Hyalellidae). Neotropica, La Plata, 14(45): 107-117.

Cervellini, P.M. 1987. Las larvas y postlarvas de crustáceos Decapoda en el estuario y las aguas marinas de Bahía Blanca (Prov. de Buenos Aires). Distribución espacial, variación estacional y su relación con los factores ambientales. Tesis doctoral, Universidad Nacional del Sur, 247 pp.

Cesar, I.I. 1985. Branchinecta achalensis sp. nov. (Crustacea: Anostraca) de la Pampa de Achala (Córdoba, República Argentina). Neotrópica, La Plata, 31(85): 89-100.

Cesar, I.I. 1987a. Branchinecta santacrucensis sp. nov. (Crustacea: Anostraca) de la Patagonia. Neotrópica, La Plata, 33(9): 75-82.

Cesar, I.I, 1987b. Branchinecta leonensis sp. nov. (Crustacea: Anostraca) de la provincia de Santa Cruz, República Argentina. Notas Museo de La Plata, 21(206): 77-86.

Cesar, I.I. 1989a. Comparative study on the resting eggs of several anostracans (Crustacea: Anostraca). Key for determination of the species based upon the egg structure and diameter. Stud. Neotrop. Fauna Environ., 24(4): 169-181.

Cesar, I.I. 1989b. Geographic distribution of the anostracans (Crustacea: Anostraca) in Argentina, (South America). Stud., Neotrop. Fauna environ., 24(4): 183-188.

Cicchino, G.N. 1972. Desarrollo post-embrionario de Notodiaptomus coniferoides (Wright, 1927) y Eucyclops neumanni (Pesta, 1927) (Crustacea: Copepoda). Physis, Buenos Aires, 31(83): 585-596.

Cicchino, G.N. 1975. Redescripción y desarrollo postembrionario de Pseudodiaptomus richardi inaequalis (Brian) con notas acerca de sus afinidades con otras especies del género (Copepoda: Pseudodiaptomidae). Physis, Buenos Aires, B34(88): 37-49.

Cicchino, G.N. y R.A. Ringuelet. 1977. Copépodos del género Antarctobiotus de Tierra del Fuego e Islas Malvinas, Rep. Argentina (Harpacticoida: Canthocamptidae). Limnobios, 1(6): 207-228

Ciechomski, J.D. y R.P. Sánchez. 1983. Relationship between ichthyoplankton 
abundance associated zooplankton biomass in the shelfwaters off Argentina. Biol. Oceanogr., New York, 3(1): 77-101.

Cohen, R.G. 1981. Notas sobre anostracos neotropicales (Crustacea). I. Branchinecta valchetana sp. nov. y redescripción de Branchinecta palustris Birabén, 1946. Physis,Buenos Aires, B40(98): 1-13.

Cohen, R.G. 1982. Nota sobre anostracos neotropicales (Crustacea). II. Branchinecta rocaensis sp. nov. Physis, Buenos Aires, B40(99): 93-98.

Cohen, R.G. 1983. Notas sobre anostracos neotropicales (Crustacea). III. Branchinecta somuncurensis y Branchinecta prima sp. nov. Physis, Buenos Aires, B41(101): 69-80.

Cohen, R.G. 1985. Notas sobre anostraca neotropicales (Crustacea). IV. Branchinecta vuriloche sp. nov. Physis, Buenos Aires, B43(104):1-6.

Cohen, R.G. 1987. Nota sobre anostracos neotropicales. V. Nuevas observaciones morfológicas sobre Branchinecta achalensis César 1986. Physis, Buenos Aires, B45(108): 13-20.

Cohen, R.G. 1991a. Anostracos de la familia Branchinectidae. Tesis doctoral, Universidad de Buenos Aires, 270 pp.

Cohen, R.G. 1991b. Sobre la presencia del palpo mandibular en ejemplares maduros de Anostraca (Crustacea: Branchiopoda). Physis, Buenos Aires, B47(112): 1-6.

Cohen, R.G. 1992. Redescription of Branchinecta granulosa Daday 1902 from South Argentina (Crustacea: Branchiopoda). Hydrobiologia, 228: 195-202.

Cohen, R.G. 1993. Nuevos aportes a la morfología y distribución de Branchinecta iheringi Lilljeborg 1889 (Crustacea: Anostraca). Physis, Buenos Aires, B48(114-115): 1-5.

Cohen, R.G. 1995. Crustacea Anostraca. In: Ecosistemas de Aguas Continentales. Metodologías para su estudio. (E.C. Lopretto y G. Tell, Dir.) Ediciones Sur, La Plata, 871-895.

Comoglio, L.I. 1994. La nutrición de los crustáceos decápodos en el Canal Beagle, con especial énfasis en centolla (Lithodes santolla) y centollón (Paralomis granulosa) y la función trófica de los mismos en el ecositema. Tesis doctoral, Universidad de Buenos Aires, 173 pp.
Comoglio, L.I., J.H. Vinuesa y G.A. Lovrich. 1990. Feeding habits of southern king crab Lithodes santolla and false southern king crab Paralomis granulosa in the Beagle Channel. Proc. Int. Symp. on King and Tanner crabs. Alaska, Anchorage, pp. 315-325.

Christoffersen, M.L. 1979. Decapod Crustacea: Alpheoida. Campagne de la Calypso au large des côtes Atlantiques de l'Amérique du Sud (1961-1962). Résultats Scientifiques des Campagnes de la Calypso, 11: 297-377.

Christoffersen, M.L. 1988. Genealogy and phylogenetic classification of the world Crangonidae (Crustacea: Caridea) with a new species and new records for the South Western Atlantic. Rev. Nordestina de Biol. Brasil, 6(1): 43-59.

Delamare, C. y C. Roland. 1963. Syncarides. Austrobathynella patagonica Delamare. Biologie de l'Amérique Australe, (Delamare Deboutteville y Rapoport, Dirs.), 2. Etudes sur la faune du sol. Paris, 55-62 pp.

Delgado de Layño, R.E. y R. Pallares. 1981. Variación estacional del contenido de lípidos en Tigriopus californicus (Backer) (Copepoda, Harpacticoida). Ecosur, Buenos Aires, 8(15): 53-64.

Díaz, A.C. y A.M. Petriella. 1988. Estudio del crecimiento del langostino Pleoticus muelleri. Rev. Latinoam. Acuic. Perú, 35: 6-12.

Dimant, B. 1991. Comportamiento exploratorio en el cangrejo Chasmagnathus granulata. Habituación y aprendizaje apetitivo. Tesis doctoral, Universidad de Buenos Aires, 97 pp.

Escofet, A. 1970. Amphipoda marinos de la Provincia de Buenos Aires. I. Bathyporelapus bisetosus sp. nov. (Gammaridea: Oedicerotidae). Neotropica, La Plata, 16(51): 101-106.

Escofet, A. 1973. Los géneros de anfípodos más comunes en el área de Mar del Plata. Clave para su reconocimiento. Contrib. Inst. Biol. Marina, Mar del Plata, 239: 22 pp.

Fenucci, J.L. 1975. Los cangrejos de la familia Pinnotheridae del litoral argentino (Crustacea, Decapoda, Brachyura). Physis Buenos Aires, 34(88): 165-184.

Fenucci, J.L., M.I. Müller y J.H. Magnaterra, 1990. Factibilidad de cría del langostino (Pleoticus muelleri). Frente Marítimo, 7(B): 103-108. 
Fenucci, J.L., M.I. Müller y A.M. Petriella,1981. Efectos de la alimentación natural y artificial en el crecimiento del camarón Artemesia longinaris Bate. Rev. Lat. Acuic. SELA, México, 1: 1-17.

Fenucci, J.L., C. Virasoro, M.B. Cousseau y E.E. Boschi. 1974. Campaña Tierra del Fuego 74, Informe preliminar. Contrib. Inst. Biol. Marina, Mar del Plata, 261: 1-37.

Fernández, N.C. 1994. Estudios sobre la biomasa de Copepoda (Crustacea) con especial énfasis en Calanoida, del Atlántico Sudoccidental. Tesis doctoral, Univ. Nacional del Mar del Plata, $91+111 \mathrm{pp}$.

Fesquet, A.E.J. 1933. Anotaciones para una monografía sobre el langostino (Pleoticus muelleri Bate). Buenos Aires. Edit. Kapeluz, 1-36.

Fesquet, A.E.J. 1941a. Descripción del mecanismo articular de los pedúnculos oculares de Artemesia longinaris Bate y de Hymenopenaeus muelleri (Bate). Rev. Holmbergia, Buenos Aires, 3(6): 64-67.

Fesquet, A.E.J. 1941b. Estudio de la morfología externa del camarón de rostro largo (Artemesia longinaris). An. Soc. Cient. Argent., Buenos Aires, 131: 60-95.

Fischbach, C. 1993. Ecología larval del langostino (Pleoticus muelleri). Algunos aspectos de la dinámica de los estadios planctónicos en el litoral bonaerense, Argentina. Frente Marítimo, 14: 101-109.

Forest, J. y M. de Saint Laurent. 1967. Crustacés Décapodes: Pagurides. Res. Scient. Campag. de la Calypso 8. Ann. Inst. Ocean. Paris, 45(2): 47-169.

Giambiagi, D. 1922. Cuatro nuevos isópodos de la Argentina. Physis, Buenos Aires, 5(20): 230-244.

Grosso, L.E. y G.L. Claps. 1984. Tercer Bogidiellidae (Crustacea: Amphipoda) de la cuenca del río Grande (Jujuy, Argentina). Neotrópica, La Plata, 30(84): 223-231.

Grosso, L.E. y R.A. Ringuelet. 1979. Fauna subterránea de las aguas dulces de la República Argentina. I. Dos nuevas especies de anfípodos del género Bogidiella. Limnobios, La Plata, 1(9): 381-394.

Guglielmo, L. y A. Ianora, (Ed.) 1995. Atlas of marine zooplankton. Straits of Magellan. Copepods. Springer Verlag., Berlin, 279 pp.
Guinot, D. y E. Macpherson. 1987. Révision dugenre Pilumnoides Lucas, 1844, avec description de quatre espèces nouvelles et création de Pilumnoidenae subfam. nov. (Crustacea Decapoda Brachyura). Bull. Mus. nat. Hist. nat. Paris, 4(9)A, 1: 211-247.

Haig, J. 1966. Porcellanid crabs (Crustacea, Anomura). Campagne de la Calypso au large descôtes Atlantiques de l'Amerique du Sud (1961-1962). Résultats Scientifiques des Campagne de la Calypso, 7: 351-358.

Haran, N. Acción de ácidos grasos dietarios sobre el crecimiento del langostino argentino Pleoticus muelleri Bate, (en prensa).

Holthuis, L.B. 1974. The lobsters of the superfamily Nephropidea of the Atlantic Ocean (Crustacea: Decapoda). Bull. Mar. Sci., 24(4): 723-884.

Holthuis, L.B. 1993. The recent genera of the Caridean and Stenopodidean shrimps (Crustacea: Decapoda) with an appendix on the order Amphionidacea. National Natuurhistorisch Museum, 328 pp.

Iorio, M.I., M.A Scelzo y E.E. Boschi. 1990. Desarrollo larval y postlarval del langostino Pleoticus muelleri Bate, 1888 (Crustacea, Decapoda, Solenoceridae). Sci. Mar., 54(4): 329-341.

Jeckel, W.H. 1990. Composición bioquímica, lipídica y en ácidos grasos del langostino Pleoticus muelleri (Bate, 1888) en relación a factores fisiológicos y ecológicos. Tesis doctoral, Universidad Nacional de Mar del Plata, 112 pp.

Jeckel, W.H, J.E. Aizpun de Moreno y V.J. Moreno. 1989. Biochemical composition, lipid classes and fatty acids in the ovary of the shrimp Pleoticus muelleri Bate. Comp. Biochem. Physiol., 92B(2): 271-276.

Lopretto, E.C. 1976. Morfología comparada de los pleópodos sexuales masculinos en los Trichodactylidae de la Argentina (Decapoda: Brachyura). Limnobios, La Plata, 1(3): 67-94.

Lopretto, E.C. 1978. Estructura exoesqueletaria y miológica del quinto par de pereiópodos del macho de la Familia Aeglidae (Crustacea, Anomura). Limnobios, La Plata, 1(8): 284-298.

Lopretto, E.C. 1980. Clave para la determinación de las especies del género Aegla de la República Argentina en base al estudio comparativo del quinto par de pereiópodos masculinos (Crustacea, Anomura, Aeglidae). Limnobios, La Plata, 1(10): 431-436. 
Lopretto, E.C. 1981. Discusión sobre la presunta subespecie de Dilocarcinus (D.) pagei (Crustacea, Brachyura, Trichodactylidae). Redescripción y referencia a su polifenismo. Physis, Buenos Aires, B39(97): 21-31.

Lopretto, E.C. 1982. Contribución a la bioecología del anfípodo dulciacuícola Hyalella pampeana Cavalieri. II. Nota preliminar sobre el desarrollo embrionario (Amphipoda, Hyalellidae). Neotropica, La Plata, 28(8): 97-102.

Lopretto, E.C. 1983a. Contribución a la bioecología del anfípodo dulciacuícola Hyalella pampeana Cavalieri. I. Comportamiento reproductivo. Limnobios, La Plata, 2(6): 371-378.

Lopretto, E.C. 1983b. Contribución a la bioecología del anfípodo dulciacuícola Hyalella pampeana Cavalieri. III. Desarrollo postembrionario. Limnobios, La Plata, 2(7): 471-490.

Lopretto, E.C. 1995. Crustacea Conchostraca. In: Ecosistemas de Aguas Continentales. Metodología para su estudio (Lopretto y Tell. Dir.) Ediciones Sur, La Plata, Argentina, pp. 897-901.

Lopretto, E.C. 1995. Crustacea Notostraca. In: Ecosistemas de Aguas Continentales. Metodología para su estudio. (E.C. Lopretto y G. Tell, Dirs.). Ediciones Sur, La Plata, Argentina, 897-901 pp.

Lopretto, E.C. y G. Tell. 1995. Ecosistemas de Aguas Continentales. Metodologías para su estudio. Ediciones Sur, La Plata, Argentina, Tomos I, II, III, 1401 pp.

Lovrich, G.A. 1991. Reproducción y crecimiento del centollón, Paralomis granulosa (Crustacea, Anomura, Lithodidae). Tesis doctoral. Universidad de Buenos Aires, 160 pp.

Lovrich, G.A. y J.H. Vinuesa. 1993. Reproductive biology of the false southern king crab (Paralomis granulosa, Lithodidae) in the Beagle Channel, Argentina. U.S. Fish. Bull., 91(4): 664-675.

Lovrich, G.A. y J.H. Vinuesa. 1995. Growth of juvenile false southern king crab Paralomis granulosa (Anomura: Lithodidae) in the Beagle Channel, Argentina. Sci. Mar., 59(1): 87-94.

Lovrich, G.A. Evolution of an unmanaged crab resource in South America: the false southern king crab Paralomis granulosa, (en prensa).
Lovrich, G.A. y J.H. Vinuesa. Reproductive strategies of two lithodids in the Beagle Channel, Argentina A complementary management tool in a changing Fisheries Int. Symp. on Biol., Manag. and Economics crabs from high lat. Habitats, Alaska, Oct.11-12 1995, (en prensa).

Macchi, G.J., M.I. Iorio y H. Christiansen. 1992. Aspectos del desove y fecundidad del langostino Pleoticus muelleri (Bate, 1888) (Crustacea, Decapoda, Solenoceridae). Rev. Biol. Mar., Valparaíso, 27(1): 43-58.

Macpherson, E. 1988. Revision of the family Lithodidae Samoulle, 1819 (Crustacea, Decapoda, Anomura) in the Atlantic Ocean. Monogr. Zool. Mar., 2: 9-153.

Mallo, J.C. 1984. Desarrollo larval y cultivo en laboratorio del camarón marino Peisos petrunkevitchi (Crustacea, Decapoda, Sergestidae). Tesis doctoral, Universidad Nacional de La Plata, 185 pp.

Mallo, J.C. 1986. Desarrollo larvario del camarón Peisos petrunkevitchi Burkenroad, 1945, en laboratorio, (Crustacea, Decapoda,Sergestidae). Biol. Pesquera, 15: 3-16.

Mallo, J.C. Respuesta a la acción de la temperatura, salinidad y contaminantes de postlarvas de Pleoticus muelleri y Penaeus paulensis (en prensa).

Mallo, J.C. y E.E. Boschi. 1982. Contribución al conocimiento del ciclo vital del camarón Peisos petrunkevitchi de la región de Mar del Plata, Argentina (Crustacea, Decapoda, Sergestidae). Physis, Buenos Aires, A41(100): 85-98.

Mallo, J.C., J.L. Fenucci, C.M. Galarza, M.F. Cautere y G. Sarlo. Cría masiva de larvas y postlarvas del langostino argentino Pleoticus muelleri Bate (Crustacea, Decapoda, Solenoceridae) (en prensa).

Manning, R.B. 1966. Stomatopod Crustacea. Resultats Scient. Camp. de la Calypso. 7. Ann. Inst. Océan. Paris, 44: 359-384.

Manning, R.B. 1990. History of the Crustacean Society, 1979-1989. J. Crust. Biol., 10(4):735-750.

Manning, R. y L.B. Holthuis. 1989. Two new genera and nine new species of geryonid crabs (Crustacea, Decapoda, Geryonidae). Proc. Biol. Soc. Wash., 102(1): 50-77. 
Manning, R.B. y D.L. Felder. 1991, Revision of the American Callianassidae (Crustacea: Decapoda: Thalassinidea). Proc. Biol. Soc. Wash., 104(4): 764-792.

Manning, R.B. y R. Lemaitre. 1993. Sergio, a new genus of ghost shrimp from the Americas (Crustacea: Decapoda: Callianassidae). Nauplius, Rio Grande, 1: 39-43.

Marcovecchio, J.E. 1988. Estudio comparativo de la distribución de los metales traza, cadmio y zinc en organismos de dos zonas estuariales de Argentina: Bahía Blanca y desembocadura del Río de la Plata. Tesis doctoral. Univ. Nacional de Mar del Plata, 212 pp.

Marcovecchio, J.E. 1994. Trace metal residues in tissues of two crustacean species from the Bahia Blanca estuary, Argentina. Environ. Monit. and Assessment, 29: 65-73.

Marschoff, E. y A. Tomo. 1984. Vertical distribution of calyptopes larvae of Euphausia superba in the Scottia Sea. In: The biology of the Antartic krill Euphausia superba. J. Crust. Biol., 4 (Special Number 1): 247-251.

Martin, J.W. y L.G. Abele. 1988. External morphology of the genus Aegla (Crustacea: Anomura: Aeglidae). Smith. Contrib. Zoology, 453: 1-46.

Mazzocchi, M.G. y A. Ianora. 1991. A faunistic study of the copepod assemblages in the Strait of Magellan. Boll. Oceanol. Teor. Appl. Trieste, 9(2-3): 163-177.

McCain, J.C. 1965. The Caprellidae (Crustacea: Amphipoda) of Virginia. Chesapeake Sci., 6(33): 190-196.

Menú-Marque, S. 1970. Desarrollo larval del cangrejo Platyxanthus crenulatus (A. MilneEdwards, 1879) en el laboratorio (Decapoda, Brachyura, Xanthidae). Physis, Buenos Aires, 29(79):477-494.

Menú-Marque, S. 1982. Thermocyclops decipiens (Kiefer, 1929), una nueva especie para la fauna argentina (Copepoda, Cyclopoida). Physis, Buenos Aires, B44(100): 41-46.

Menzies, R.J. 1962. The isopods of abyssal depths in the Atlantic Ocean. Vema Res. Ser., 1: 79-206.

Moguilevsky, A. y R. Whatley. 1995. Crustacea Ostracoda. In: Ecosistemas de aguas continentales. Metodologías para su estudio (E.C. Lopretoy G. Tell, Dir.), Ediciones Sur, La Plata, Argentina. 973-999 pp.
Monserrat, J.M., E.M. Rodriguez y R.J. Lombardo. 1991. Effects of salinity on the toxicity of parathion to the estuarine crab Chasmagnathus granulata (Decapoda, Grapsidae). Bull. Environ. Contam. Toxicol., 46: 569-575.

Montú, M. 1977. Eufáusidos de la plataforma argentina y adyacencias. I. Distribución estacional en el sector patagónico. Ecosur, 4(8): 187-225.

Moreno, V.J. 1977. Composición lipídica y biosíntesis de ácidos grasos de organismos del fitoplancton y zooplancton marino Phaedactylum tricornutum y Paracalanus parvus respectivamente. Tesis doctoral, Universidad Nacional de La Plata, 165 pp.

Olivier, R.S. 1954a. Cladóceros marinos de la Argentina. Notas del Museo La Plata, Zool., 17(151): 157-166.

Olivier, R.S. 1954b. Una nueva especie del género Moina (Crust. Cladocera). Notas Museo La Plata, Zool., 17(147): 67-70.

Olivier, S.R. 1962. Los cladóceros argentinos. Con clave de las especies, notas biológicas y distribución geográfica. Revista del Museo La Plata, (NS) Zool., 7: 173-269.

Paggi, J.C. 1994. Biodiversidad del zooplancton en los ecosistemas acuáticos continentales de la región neotropical: Revisión de las especies del género Notodiaptomus Kiefer (Copepoda, Calanoida). Tesis de Magister en Ecología Acuática Continental, Universidad Nacional del Litoral, $121 \mathrm{pp}$.

Paggi, J.C. 1995. Crustacea Cladocera. In: Ecosistemas de Aguas Continentales, Metodologías para su estudio. (E.C. Lopreto y G.Tell, Dirs.) Edicione Sur La Plata, Argentina, 909-951 pp.

Pallares, R.E. 1968. Copépodos marinos de la Ría Deseado (Santa Cruz, Argentina). Serv. Hidrog. Naval, Buenos Aires, H1024: 125 pp.

Pallares, R.E. y M.A. Hall. 1974. Análisis bioestadístico-ecológico de la fauna de copépodos asociados a los bosques de Macrocystis pyrifera. Physis, Buenos Aires, 33(87): 409-432.

Pérez Farfante, I. 1977. American solenocerid shrimps of the genera Hymenopenaeus, Haliporoides, Pleoticus, Hadropenaeus new genus, and Mesopenaeus new genus. U.S. Fish. Bull., 75(2): 261-346. 
Pérez Farfante, I. 1988. Illustrated key to penaeoid shrimps of commerce in the Americas. NOAA Tech. Rep. NMFS 64: 1-32.

Petriella. A.M. 1984. Estudio del ciclo de muda del camarón Artemesia longinaris Bate. I. Setogénesis. Physis, Buenos Aires, A42(103): 93-100.

Petriella, A.M. 1986. Estudio del ciclo de muda del camarón Artemesia longinaris Bate. II. Crecimiento y frecuencia de muda. Rev. Lat. Acuic., 29: 11-21.

Petriella, A.M. 1987. Estudio del ciclo de muda del camarón Artemesia longinaris: descripción, crecimiento y relación con el colesterol. Tesis doctoral, Universidad de Buenos Aires, 176 pp.

Petriella, A.M., M.I. Müller, J.L. Fenucci y M.B. Saenz. 1984. Influence of the dietary fatty acids and cholesterol on the growth and survival of the argentine prawn Artemesia longinaris Bate.Aquaculture, 37: 11-20.

Petriella, A.M. y A.C. Díaz. 1987. Influence of eyestalk ablation on moulting frequency and gonadal maturation of the argentine prawn Artemesia longinaris Bate. J. Aquacult. Trop., 2(1): 17-24.

Petriella, A.M. y R.J. Bridi. 1992. Variaciones estacionales del ciclo de muda y la maduración ovárica del camarón Artemesia longinaris Bate. Frente Marítimo, 11(A): 85-92.

Ramírez, F.C. 1966. Copépodos calanoideos marinos del area de Mar del Plata, con la descripción de Pontella marplatensis n. sp. Bol. Inst. Biol. Marina, Mar del Plata, 11: 1-24.

Ramírez, F.C. 1967. Ostrácodos de lagunas de la provincia de Buenos Aires. Rev. Mus. La Plata (N.S.), Zool., 10: 5-54.

Ramírez, F.C. 1969. Copépodos planctónicos del sector bonaerense del Atlántico Sudoccidental. Proy. Des. Pesquero, Mar del Plata, Inf. Téc., 10: 1-116.

Ramírez, F.C. 1970. Copépodos planctónicos del sector patagónico. Physis, Buenos Aires, 29(79): 473-476.

Ramírez, F.C. 1971a. Copépodos planctónicos de los sectores bonaerense y norpatagónico. Rev. Mus. La Plata (NS), 11(97): 73-94.

Ramírez, F.C. 1971b. Eufáusidos de algunos sectores del Atlántico Sudoccidental. Physis, Buenos Aires, 30(81): 385-405.
Ramírez, F.C. 1973. Eufáusidos de la campaña oceanográfica «Walther Herwig». Physis, Buenos Aires, 32(84): 105-114.

Ramírez, F.C. 1975. Sobre una nueva especie del género Kroyerina Wilson, 1952 (Crustacea: Copepoda). Neotropica, La Plata, 21(64): 37-41.

Ramírez, F.C. 1976. Resultados planctológicos de la campaña «Oceantar I»: Copépodos. Cont. Inst. Antártico Arg., 196: 1-49.

Ramírez, F.C. 1977. Planktonic indicators of Argentina shelf and adjacent areas. Proc. Symp. warm water zoopl., Spec. Publ., UNESCO-NIO, Goa, India: 65-68.

Ramírez, F.C. 1981a. Cladocera. In: D. Boltovskoy (ed.), Atlas del Zooplancton del Atlántico Sudoccidental, y Métodos de Trabajo con el Zooplancton Marino, INIDEP Publicación especial, 533-542 pp.

Ramírez, F.C. 1981b. Zooplancton y producción secundaria. Parte I. Distribución y variación estacional de los copépodos. Contrib. INIDEP, Mar del Plata, 383: 202-232.

Ramírez, F.C. y A. Escofet. 1973. Sobre la presencia de Hemicyclops subadhaerens Gooding, 1960 (Copepoda, Clausidiidae) asociado con Callianassa sp. (Decapoda, Thalassinidea) del golfo San Matías, Río Negro, Argentina. Physis, Buenos Aires, 32(85): 301-310.

Ramírez, F.C. y P. de Vreese. 1974. Taxonomía y distribución de los cladóceros (Crustacea, Phylopoda) de un sector de la plataforma bonaerense y adyacencias. Physis, Buenos Aires, A33(87): 511-526.

Ramírez, F.C. y C. Dato. 1983. Seasonal changes in population structure and gonadal development of three euphausid species. Oceanol. Acta, 6(4): 427-433.

Ramírez, F.C., J.M. Orensanz, E. Dinofrio y P. de Vreese. 1974. Resultado del análisis del zooplancton de la campaña «Oceantar I», con especial referencia a los cópepodos, quetognatos, poliquetos y sálpidos. In: $2^{\circ}$ Reunión del Grupo Internacional de Coordinación de los océanos australes, Buenos Aires, (CADO-CONICET), pp. 57-63.

Ramírez, F.C. y G.M. Pérez. 1981a. Observaciones sobre el desarrollo postnaupliar, estructura poblacional y ciclo reproductivo del ostrácodo planctónico Conchoecia serrulata Claus. Physis, Buenos Aires, A40(98): 15-32. 
Ramírez, F.C. y G.M. Pérez. 1981b. Variación cuali-cuantitativa del contenido alimentario del ostrácodo planctónico Conchoecia serrulata Claus. Neotropica, La Plata, 27(28): 113-119.

Ramírez, F.C. y M.D. Viñas. 1985. Hyperiid amphipods found in Argentine shelf waters. Physis, Buenos Aires, A43(104): 25-37.

Ramírez, F.C. y C. Dato. 1989. Observations on parasitism by Thalassomyces fagei on three euphausid species in southern atlantic waters. Oceanol. Acta, 12(1): 95-97.

Ramner, W. 1933. Die Cladoceren der «Meteor» Expedition. Wiss. Ergeb. Deutch. Atlant. Exp. Forsch Vremess. Meteor, 1925-1927, Berlin-Leipzig, 12: 111-121.

Rathbun, M.J. 1918. The grapsoid crabs of America. U.S. Nat. Mus. Washington, Bull. 97: 1-461.

Rathbun, M.J. 1925. The spider crabs of America. U.S. Nat. Museum, Washington. Bull., 129: 1-613.

Rathbun, M.J. 1930. The cancroid crabs of America of the family Euryalidae, Portunidea, Atelecyclidae, Cancridae and Xanthidae. U.S. Nat. Museum, Washington, Bull. 152: 1-609.

Rathbun, M.J. 1937. The oxystomatous and allied crabs of America. U.S. Nat. Museum, Washington, Bull. 166, 1-278.

Reca, A.R. 1970. Oniscoideos argentinos. I. Sobre la posición sistemática de Philoscia argentina Giambiagi, 1939 (Crustacea, Isopoda). Physis, Buenos Aires, 29(79): 423-429.

Reca, A.R. 1972. Oniscoideos argentinos. II. Tres especies de isópodos terrestres de la costa marítima bonaerense (Crustacea, Isopoda). Physis, Buenos Aires, 31(83): 405-410.

Reca, A.R. 1973. Oniscoideos argentinos. III Aporte al conocimiento de la subfamilia Bathytropinae (Isopoda, Oniscidae). Physis, Buenos Aires, 32(84): 93-99.

Ringuelet, R.A. 1943. Revisión de los argúlidos argentinos (Crustacea, Branchiura) con el catálogo de las especies neotropicales. Rev. Mus. La Plata (NS), Zool., 3: 43-99.

Ringuelet, R.A. 1945. Parabrachiella spinicephala nueva especie de copépodo parásito. Notas Mus. La Plata, Zool., 86: 129-133.
Ringuelet, R.A. 1948. Argúlidos del Museo de La Plata. Rev. Mus. La Plata (NS), Zool., 5: 281-296.

Ringuelet, R.A. 1948a. Una nueva Aegla del nordeste argentino. Not. Mus., La Plata, 13(111): 203-208.

Ringuelet, R.A. 1948b. Los cangrejos argentinos del género Aegla de Cuyo y la Patagonia. Rev. Mus. de La Plata (NS), Zool., 5(34): 297-347.

Ringuelet, R.A. 1949a. Los anomuros del género Aegla del noroeste de la República Argentina. Rev.Mus. La Plata (NS), Zool., 6(36): 1-45.

Ringuelet, R.A. 1949b. Camarones y cangrejos de la zona de Goya (Sergestidae, Palaemonidae y Trichodactylidae) Not. Mus. de La Plata, Zool., 14(119): 79-109.

Ringuelet, R.A. 1958a. Primeros datos ecológicos sobre copépodos dulceacuícolas de la República Argentina. Physis, Buenos Aires, 21(60): 14-31.

Ringuelet, R.A. 1958b. Los crustáceos copépodos de las aguas continentales de la República Argentina. Contrib. Cient. Fac. Cienc. Exactas y Nat.Buenos Aires, Zool. 1(2): 1-92.

Ringuelet, R.A. 1961. Panorama zoológico argentino. Invertebrados excepto insectos. SesionesCientíficas de Zoología. Tucumán 6-11 nov. 1960. Physis, Buenos Aires, 22(63): 21-33.

Ringuelet, R.A. 1963. Hallazgo de Callinectes sapidus acutidens Rathbun en la ribera occidental del Río de la Plata (Crustacea, Brachyura, Portunidae). Physis, Buenos Aires, 24(67): 86.

Ringuelet, R.A. 1968. Biogéographie des copépodes d'eau douce de l'Argentine. Biologie de l'Amérique Australe. (Delamare Deboutteville y Rapoport, Dirs.) Paris, 4: 261267.

Ringuelet, R.A. y A. Martínez. 1967. Notas sobre diaptómidos argentinos (Crustacea, Copepoda). Physis, Buenos Aires, 26(73): 411-420.

Roccatagliata, D.C. 1990. Sistemática y distribución de los Bodotriidae (Cumacea, Crustacea) del Atlántico Sudamericano y aportes al conocimiento de la biología del orden Cumacea. Tesis doctoral, 261-267.Universidad de Buenos Aires, $243 \mathrm{pp}$.

Roccatagliata, D. 1993. On two southwest species of the genus Leptocuma Sars, 1873 (Crustacea: Cumacea). J. Natural History, London, 17: 299-312. 
Rodríguez, E.M. y O.A. Amin. 1991. Acute toxicity of parathion and 2,4D to larval and juvenile stages of Chasmagnathus granulata (Decapoda, Brachyura). Bull. Environ. Contam. Toxicol., 47: 634-640.

Rodríguez, E.M. y J.M. Monserrat. 1991. Acute and chronic effects of parathion and 2,4D on the oxigen consuption of Chasmagnathus granulata (Decapoda: Brachyura). APPTLA, 41(2): 201 210.

Rodríguez, E.M. y R.J. Lombardo. 1991. Acute toxicity of parathion and 2,4D to estuarine adult crabs. Bull. Environ. Contam. Toxicol, 46: 576 -582 .

Rodríguez, E.M. y A. Pisano. 1993. Effects oparathion and 2,4D to the eggs incubation and larvae hatching in Chasmagnathus granulata (Decapoda: Brachyura).

Rodríguez, E.M., J.M. Monserrat y O.A. Amin.1992. Chronic toxicity of ethyl parathion and isobutoxyethalnol ester of 2,4 dichloropheno- xyacetic acid to estuarine juvenile and adult crabs. Arch. Environ., Contam. Toxicol., 22: 140-145.

Rodríguez, G. 1992. The freshwater crabs ofAmerica. family Trichodactylidae and supplement to the family Pseudothelphusidae. Inst. Fran. Recher. Develop. Coop. Colec. Faune Tropicale, 31: 1-189.

Rouch, R. 1962. Harpacticoides (Crustacés copépodes d'Amerique du Sud). Biol. de l'Amer. australe. Etudes sur la faune du sol, (Delamare, Deboutteville y Rapoport, Dirs.). Paris, 1: 237-280.

Roux, A. y C. Bremec. Comunidades bentónicas relevadas en tres transecciones realizadas frente al Río de la Plata, Mar del Plata y Península Valdés, Argentina. INIDEP Informe Técnico, Mar del Plata, (en prensa).

Roux, A., M. Fernández y C. Bremec. 1995. Estudio preliminar de las comunidades bentónicas de los fondos de pesca de langostino patagónico del golfo San Jorge (Argentina). Ciencias Marinas, 21(3): 295-310.

Sabatini, M.E. 1987. Fito y zooplancton de un sector de la Bahía Blanca: especies dominantes, stan-ding stock y estimación de la producción, con particular referencia a Acartia tonsa Dana, 1849 (Copepoda, Calanoida). Tesis doctoral, Universidad Nacional de Sur, 164 pp.
Sarlo, E.G. 1995. Desarrollo embrionario de Pleoticus muelleri Bate, 1888 (Crustacea, Decapoda, Solenoceridae), en condiciones de laboratorio. Efecto de la temperatura. Tesis de licenciatura. Facult. de Cienc. Exactas y Nat., Universidad Nacional de Mar del Plata, 43 pp.

Scelzo, M.A. 1973. Lista de los crustáceos decápodos anomuros obtenidos en 1966 por la expedición Walther Herwig en el Atlántico Sur y depositada en las colecciones del Instituto de Biología Marina. Physis, Buenos Aires, A32(84): 161-174.

Scelzo, M.A. y E.E. Boschi. 1973. Aportes al conocimiento de la distribución geográfica de los crustáceos decápodos Anomura del Atlántico Sudoccidental, frente a las costas argentinas.Trab.V Cong. Lat. de Zoología Montevideo, 1: 204-216.

Scelzo, M.A. y E.E. Boschi. 1975. Cultivo del langostino Hymenopenaeus muelleri (Crustacea, Decapoda, Penaeidae). Physis, Buenos Aires, 34(88): 193-197.

Scelzo, M.A., J.L. Fenucci y E.E. Boschi. 1974. Resultados preliminares sobre la biología pesquera de la centolla Lithodes antarcticus en el Mar Argentino. CARPAS, Montevideo, 6/74/ Tec., 4: 1-13.

Scelzo, M.A. y A. Valentini. 1974. Presencia de Geryon quinquedens Smith, en aguas del Océano Atlántico Sudoccidental (Decapoda, Brachyura, Geryonidae). Physis, Buenos Aires, A33(87): 557-567.

Schmitt, W.L. 1942a. A new species of sand-bug, Blepharipoda doelloi of Argentina. Smith. Misc. Collec., CI(18): 1-10.

Schmitt, W.L. 1942b. The species of Aegla, endemic South America freshwater crustacea. Proc. U.S. Nat. Mus., 91(3132): 431-520.

Semenov, V.N. 1972. Tipos de habitat de los invertebrados bentónicos y biogeografía de las aguas templadas de América del Sur. Proc. Vniro, 72: 120-152 (en ruso).

Spivak, E.D. 1987. Estudio sobre la muda y el crecimiento de Cyrtograpsus angulatus. Tesis doctoral, Universidad de Buenos Aires, 147 pp.

Spivak, E.D. 1988. Moult and growth inCyrtograpsus angulatus Dana (Decapoda, Brachyura). J. Nat. History, London, 22: 617-629. 
Spivak, E.D. 1990. Limb regeneration in a common South-American littoral crab Cyrtograpsus angulatus. J. Nat. History, London 24: 393-402.

Spivak, E.D., M.A. Gavio y C.E. Navarro. 1991. Life history and structure of the world's southermost Uca population: Uca uruguayensis (Crustacea, Brachyura) in Mar Chiquita lagoon (Argentina). Bull. Mar. Science, 48(3): 679-688.

Spivak, E.D. y M.A. Politis. 1989. High incidence of limb autotomy in a crab populations from a coastal lagoon in the province of Buenos Aires, Argentina. Can. J. Zool., 67: 1976-1985.

Stephenson, W. y M. Rees. 1968. A revision of the genus Ovalipes Rathbun, 1898 (Crustacea, Decapoda, Portunidae). Rec. Aust. Mus., 27(2): 213-261.

Taberner, R. 1988. Sobre las especies de agua dulce de la familia Sphaeromatidae (Isopoda, Flabellifera) I: Basphaeroma rhombofrontale (Giambiagi, 1922). Physis, Buenos Aires, B46(110): 21-27.

Tablado, A. y J. López. 1995. Host-parasite relationships between the mussel, Mytilus edulis L. and the pea crab Tumidotheres maculatus (Say) in the Southwestern Atlantic. J. Shellfish. Res., 14(2): 417-423.

Takeda, M. 1986. Crustacea. In: Important fishes trawled off Argentina. Nakamura (ed.), Japan Mar. Fish. Resour. Res. Center, 318-343 pp.

Torti, M.R. y E.E. Boschi. 1973. Nuevos aportes al conocimiento de los crustáceos decápodos Caridea del género Campylonotus Bate, 1888. Physis, Buenos Aires, A32(84): 65-84.

Truesdale, F. 1993. History of carcinology.Crustacean Issues 8, A.A. Balkema, Rotterdam/Brookfield., 445 pp.

Recibido el 24 de junio de 1996.

Aceptado el 15 de septiembre de 1996.
Vandel, A. 1963. Isopodes terrestres recueillis en Amérique du Sud par Claude Delamare Deboutteville. Biol. de L'Amerq. Austral, (Delamare Deboutteville y Rapoport, Dirs.) Paris, 2: 63-100.

Vinuesa, J.H. 1982. Biología de la reproducción y el desarrollo embrionario y larval de la centolla, Lithodes antarcticus Jacquinot, en el Canal Beagle, Tierra del Fuego. Tesis doctoral, Fac. C.E. y Nat.Universidad de Buenos Aires, 144 pp.

Vinuesa, J.H. 1984. Sistema reproductor, ciclo y madurez gonadal de la centolla (Lithodes antarcticus) del Canal Beagle. Contrib. INIDEP, Mar del Plata, 441: 73-95.

Vinuesa, J.H. 1985. Differential aspects of the southern king crab (Lithodes antarcticus) in two latitudinally separated locations. Proc. Int. King Crab Symp., Alaska, Anchorage, 267-279.

Vinuesa, J.H., G.A. Lovrich y L.I. Comoglio. 1989. Temperature-salinity effects on the development of false southern king crab (Paralomis granulosa). Thalassa, 7: 51-56.

Williams, A.B. 1974. The swimming crabs of the genus Callinectes (Decapoda: Portunidae). U.S. Fish. Bull., 72(3): 685-798.

Wright, S. 1927. A revision of the South American species of Diaptomus. Trans. Amer. Micr. Soc., 46(2): 73-121.

Wright, S. 1938. Distribuicäo geographica das species de Diaptomus na America do Sul. Livro Jub. Prof. Travassos, 3: 561-566.

Wright, S. 1939. Algunas especies del género Diaptomus halladas en la Repúblic Argentina. Physis, Buenos Aires, 17(48): 645-649.

Zarenkov, N.A. y V.N. Semenov. 1972. A new species of the genus Nephropides from the South West Atlantic. Zool. J. Moscow, 51: 599-601. 